\title{
Flux-balance equations for linear momentum and center-of-mass position of self-gravitating post-Newtonian systems
}

\author{
Luc BlANCHET* and Guillaume FAYE ${ }^{\dagger}$ \\ $\mathcal{G} \mathbb{R} \varepsilon \mathbb{C O}$, Institut d'Astrophysique de Paris, \\ UMR 7095, CNRS, Sorbonne Université, \\ 98 bis boulevard Arago, 75014 Paris, France
}

(Dated: December 19, 2018)

\begin{abstract}
We revisit the problem of flux-balance equations for isolated post-Newtonian matter systems due to the emission of gravitational waves. In particular we show by a local derivation confined to the system, using the expression of radiation-reaction forces up to the 3.5PN order, that not only the energy, angular momentum and linear momentum of the system, but also the position of its center of mass, obey some (non-trivial) flux-balance equations. The balance equation for the center-of-mass position completes the description of the secular evolution by gravitational waves of relativistic post-Newtonian isolated matter systems. We then confirm this result by a direct computation of the gravitational-wave fluxes at future null infinity, obtaining the full multipolemoment expansion of the flux associated with the center-of-mass position (probably new with this paper), and rederiving as well the known multipole-moment expansions of the fluxes of energy, angular momentum and linear momentum. We also check our analysis by a direct calculation of radiation-reaction effects in the case of compact binary systems.
\end{abstract}

*Electronic address: luc.blanchet@iap.fr

${ }^{\dagger}$ Electronic address: guillaume.faye@iap.fr 


\section{INTRODUCTION AND MOTIVATION}

Flux-balance equations and gravitational radiation reaction play a major role in gravitational physics since the discovery of the binary pulsar in 1974 [1]. At that time, the "radiation-reaction controversy" [2,3] aimed at reconciling the effect of radiation-reaction forces [4-9] on the orbit of the binary pulsar with the expected gravitational-wave fluxes for energy and angular momentum [10-15]. Since then the controversy was resolved [16, 17] and we know now that the flux-balance equations are correct, at least for extended fluid systems in an approximate "post-Newtonian" sense [18-22]. ${ }^{1}$ Nowadays, the balance equations are used for building accurate gravitational-wave templates for the data analysis of inspiralling compact binaries, since they permit to compute the evolution of the orbital phase and frequency of inspiralling compact binaries prior their final merger, i.e., the famous "chirp" of gravitational waves [23, 24].

At leading order, corresponding either to $2.5 \mathrm{PN}$ order in the equations of motion or Newtonian order in the radiation field, the energy balance is given by the Einstein quadrupole formula $[10,11]$

$$
\frac{\mathrm{d} E}{\mathrm{~d} t}=-\frac{G}{5 c^{5}} \stackrel{(3)}{I}_{i j} \stackrel{(3)}{I}_{i j}+\mathcal{O}\left(\frac{1}{c^{7}}\right)
$$

where $I_{i j}$ is the mass-type quadrupole moment of the source, the superscript $(n)$ denotes time derivatives, and $E$ in the left-hand side (LHS) is the total mechanical energy of the system. At this order $E$ is just the constant rest-mass energy plus the Newtonian mechanical energy. However, there is also a contribution to $E$ at the $2.5 \mathrm{PN}$ order, which comes from the right-hand side (RHS) of the balance equation, when one decides to write the energy flux into "canonical" form, i.e., the form of the familiar Einstein quadrupole formula. Indeed, a total time derivative appears, in the process of transforming the RHS, and is transferred to the LHS of the balance equation. This derivative represents the analogue of the Schott term in electromagnetism [25]. Note that while the flux in the RHS of (1.1) is gauge invariant, the Schott term depends on the assumed gauge used to express the radiationreaction force $[26,27]$. As is well known, such $2.5 \mathrm{PN}$ term being in the form of a total time derivative, is negligible with respect to the flux entering the RHS in the adiabatic approximation, i.e., when averaged over an orbital scale much smaller than the radiationreaction time scale. The balance equation for angular momentum reads similarly at the leading order [28, 29]

$$
\frac{\mathrm{d} J_{i}}{\mathrm{~d} t}=-\frac{2 G}{5 c^{5}} \varepsilon_{i j k} \stackrel{(2)}{I_{j l}} \stackrel{(3)}{I}_{k l}+\mathcal{O}\left(\frac{1}{c^{7}}\right)
$$

where $J_{i j}$ is the current-type quadrupole moment of the source. Again there is in the LHS a total time derivative (Schott like term) at $2.5 \mathrm{PN}$ order which is taken into account by a definition of the angular momentum $J_{i}$. The flux-balance equations for energy and angular momentum (1.1)-(1.2) can be combined to compute the secular evolution (on a radiationreaction time scale) of eccentric compact binaries $[12,13]$.

Concerning the linear momentum, the effect is subdominant as it appears at order 3.5PN rather than $2.5 \mathrm{PN}$. Indeed, the leading radiation-reaction force at $2.5 \mathrm{PN}$ order integrates to

\footnotetext{
${ }^{1}$ Following the usual post-Newtonian terminology, a term $\sim(v / c)^{n}$ is said to be of order $n$ PN. Keep in mind that there is always a difference of $2.5 \mathrm{PN}$ order between the equations of motion and the gravitational radiation field, which corresponds to the order $(v / c)^{5}$ of radiation-reaction forces.
} 
zero over the system and there is no net force at that order. Including the radiation reaction at the next 3.5PN order, one obtains the balance equation for linear momentum as [28-32]

$$
\frac{\mathrm{d} P_{i}}{\mathrm{~d} t}=-\frac{G}{c^{7}}\left[\frac{2}{63} \stackrel{(4)}{I}_{i j k} \stackrel{(3)}{I_{j k}}+\frac{16}{45} \varepsilon_{i j k} \stackrel{(3)}{I_{j l}} \stackrel{(3)}{J}_{k l}\right]+\mathcal{O}\left(\frac{1}{c^{9}}\right)
$$

where the RHS results from a coupling between the mass octupole moment $I_{i j k}$ and the mass quadrupole, as well as a coupling between the mass and current quadrupoles. The main application of the flux-balance equation for linear momentum is the estimation of the total recoil of the system by gravitational waves. The recoil velocity (or kick) of the black hole formed by the merger of two black holes has been computed by post-Newtonian methods [33-38], perturbation methods [39-42], as well as using numerical relativity [43-46].

The quantities $E, J_{i}$ and $P_{i}$ in Eqs. (1.1)-(1.3) can be referred to as the "Bondi like" quantities $[47,48]$, that secularly evolve by gravitational-radiation emission. By integrating the fluxes in the RHS and transfering these to the LHS, one can obtain the conserved invariants for the total matter system + gravitational waves and which can be referred to as "ADM like" quantities [49]. For a conservative system (for instance, stationary), there are ten Noetherian conserved invariants associated with the ten symmetries of the Poincaré group. In addition to $E, J_{i}$ and $P_{i}$, there is also the initial "position" of the center of mass, say $Z_{i}=G_{i}-P_{i} t$, where $G_{i}$ denotes the position of the center of mass multiplied by the total (constant) mass. The conservation of $Z_{i}$ is due to the invariance of the dynamics under Lorentz boosts. For self-gravitating systems, $G_{i}$ coincides with the mass-type dipole moment $I_{i}$. When gravitational-wave emission is turned on, the energy, angular momentum and linear momentum obey the balance or evolution equations (1.1)-(1.3), and we expect that the center-of-mass position should also obey a similar evolution equation.

In the present paper we prove that the flux-balance equation for the center-of-mass position is, as for (1.3), a subdominant 3.5PN effect, and involves in the flux just a coupling between the mass octupole moment and the mass quadrupole, without contribution from the current moment at the lowest order. We find

$$
\frac{\mathrm{d} G_{i}}{\mathrm{~d} t}=P_{i}-\frac{2 G}{21 c^{7}} \stackrel{(3)}{I}_{i j k} \stackrel{(3)}{I}_{j k}+\mathcal{O}\left(\frac{1}{c^{9}}\right) .
$$

Together with (1.1)-(1.3), the law (1.4) completes the description of the secular evolution by gravitational waves of relativistic isolated systems. It can be rephrased in an equivalent way by saying that the initial position $K_{i}$ of the center of mass obeys

$$
\frac{\mathrm{d} K_{i}}{\mathrm{~d} t}+t \frac{\mathrm{d} P_{i}}{\mathrm{~d} t}=-\frac{2 G}{21 c^{7}} \stackrel{(3)}{I}_{i j k} \stackrel{(3)}{I}_{j k}+\mathcal{O}\left(\frac{1}{c^{9}}\right) .
$$

The formulas (1.4) or (1.5) do not appear in standard text-books on general relativity and gravitational waves such as [11, 50-54], nor in review articles like [23, 24, 55-57]. An integral expression of the relevant flux was, however, already mentioned as a particular term in the mass dipole moment obtained by matching between the near zone and the far zone (see Eqs. (3.46) in [21] and (2.15) in [22]). Moreover, a flux-balance equation for the center of mass is given in general form by Eq. (6.35) of [54], and the flux of center-of-mass position has been computed numerically in [58].

In a recent paper, Kozameh et al. [59] (see also [60]) investigated the flux-balance equations for general isolated sources based on the asymptotic properties of the radiation field and 
Weyl scalars. They obtained notably the flux of mass-type dipole moment, equivalent to our center-of-mass position. From Eqs. (31) and (41)-(44) in [59], we see that the leading-order contribution to the flux as found there is in complete agreement with our result (1.4), modulo a total time derivative. Furthermore, Ref. [59] gives the next-to-leading-order contribution, involving the current type quadrupole and octupole moments, which is also in agreement with the corresponding term in the general multipole expansion obtained in Sec. IV.

In another interesting recent work, Nichols [61] derived related expressions for the flux of center-of-mass position, which he refers to as the CM angular momentum but is really, like for us, the Noetherian invariant associated with the boost symmetry of the system. The calculations in [61] are performed at future null infinity using the Bondi-Sachs formalism in the general case, as well as in the leading PN approximation, and are motivated by the study of the memory effect induced by changes in the multipole moments parametrizing the $\mathrm{CM}$ angular momentum. More work should be done in order to compare the results of the present paper to those of Ref. [61].

The fluxes of energy, angular momentum and linear momentum are known as full multipole-moment series expansions, formally extending Eqs. (1.1)-(1.3) up to any multipolar order [29]. The multipole expansions are given in terms of the so-called "radiative" multipole moments, which parametrize the waveform at infinity. These multipole expansions are thus exact by definition, although the radiative moments are not directly connected to the source. In the present paper, we also perform the flux computations and rederive the known multipole expansions for energy, angular momentum and linear momentum up to any multipolar order, but we adopt a different strategy and express the fluxes in terms of appropriate "source" multipole moments that are known as explicit integrals over the matter source. The prize we have to pay is that our calculation is valid only at the leading postMinkowskian order, i.e., the dominant order in $G$. By the same method, we then obtain the complete multipole expansion of the flux associated with the position of the center of mass, thus generalizing Eq. (1.4) to all multipolar contributions in terms of the source multipole moments.

The plan of this paper is as follows. In Sec. II, we recall from previous works the expression in a specific gauge of the radiation-reaction force for extended isolated systems to 3.5PN order. In Sec. III we use that expression to derive the flux-balance equations for all invariant quantities and, in particular, that for the center-of-mass position to the lowest order, proving Eq. (1.4). In Sec. IV, we perform a direct flux calculation at future null infinity for all the invariants, yielding the multipole expansions generalizing the lowest order results (1.1)-(1.4) to any multipolar order (at dominant order in $G$ ). Finally in Sec. V, we reconfirm the results for the linear momentum and center-of-mass position by working out the 3.5PN harmoniccoordinate radiation-reaction force in the case of compact binary systems. The paper ends in Sec. VI with a discussion on the meaning of the results and a short conclusion.

\section{GRAVITATIONAL RADIATION REACTION TO 3.5PN ORDER}

The first derivation we propose is valid for a general isolated (compact-support) postNewtonian matter system. It is based on a specific expression of the radiation-reaction force at the 3.5PN order, defined in a particular gauge which is an appropriate extension of the Burke-Thorne gauge $[5,6]$ for the lowest-order radiation reaction. The $1 \mathrm{PN}$ radiation reaction in this gauge has been obtained in [21, 22]. In fact, the present calculation will be the continuation of the paper [22]. The radiation-reaction effects are entirely specified 
once we give the metric, which admits the following components, accurate to order 3.5PN concerning radiation-reaction (dissipative) effects:

$$
\begin{aligned}
& g_{00}=-1+\frac{2 \mathcal{V}}{c^{2}}-\frac{2 \mathcal{V}^{2}}{c^{4}}+\frac{1}{c^{6}} g_{60}+\frac{1}{c^{8}} g_{80}+\mathcal{O}\left(\frac{1}{c^{10}}\right), \\
& g_{0 i}=-\frac{4 \mathcal{V}_{i}}{c^{3}}+\frac{1}{c^{5}} g_{0 i}+\frac{1}{c^{7}} g_{0 i}+\mathcal{O}\left(\frac{1}{c^{9}}\right), \\
& g_{i j}=\delta_{i j}\left(1+\frac{2 \mathcal{V}}{c^{2}}\right)+\frac{4}{c^{4}}\left(W_{i j}-\delta_{i j} W_{k k}\right)+\frac{1}{c^{6}} g_{6}+\mathcal{O}\left(\frac{1}{c^{8}}\right) .
\end{aligned}
$$

Concerning conservative effects, this metric is only accurate to $1 \mathrm{PN}$ order, but notice the term of order $1 / c^{4}$ in the spatial metric $g_{i j}$, which is included for completeness and is really of order $2 \mathrm{PN}$ for the motion of massive particles and $1 \mathrm{PN}$ for the motion of photons. This term is important to control the energy of the system at 1PN order or, equivalently, the total mass up to $2 \mathrm{PN}$ order, see [22]. The coefficients ${ }_{n} g_{\mu \nu}$ in (2.1) represent some $2 \mathrm{PN}$ and $3 \mathrm{PN}$ conservative effects, uncontrolled at this stage, but which will not contribute to the present calculation. The metric is characterized by a scalar potential $\mathcal{V}$, a vector potential $\mathcal{V}_{i}$, and a tensorial one $W_{i j}$. These potentials are generated by the matter stress-energy tensor $T^{\mu \nu}$ of the compact-support source through the effective mass, current and stress densities:

$$
\sigma=\frac{T^{00}+T^{i i}}{c^{2}}, \quad \sigma_{i}=\frac{T^{0 i}}{c}, \quad \sigma_{i j}=T^{i j}
$$

with $T^{i i}=\delta_{i j} T^{i j}$. The potentials $\mathcal{V}_{\mu}=\left(\mathcal{V}, \mathcal{V}_{i}\right)$ contain a conservative part and a radiationreaction part, hence we pose

$$
\mathcal{V}_{\mu}=V_{\mu}^{\mathrm{sym}}+V_{\mu}^{\mathrm{reac}} .
$$

For the present purpose, the conservative part is defined by the "symmetric" propagator acting on the matter source proportional to $\sigma_{\mu}=\left(\sigma, \sigma_{i}\right)$, i.e.,

$$
V_{\mu}^{\mathrm{sym}}=\square_{\mathrm{sym}}^{-1}\left(-4 \pi \sigma_{\mu}\right)=\sum_{k=0}^{+\infty}\left(\frac{\partial}{c \partial t}\right)^{2 k} \Delta^{-k-1}\left(-4 \pi \sigma_{\mu}\right) .
$$

Strictly speaking, such definition does not yield a purely conservative quantity since the matter stress-energy tensor (2.2) depends on the metric (2.1) and, thus, does contain some radiation-reaction contributions. With this caveat in mind, we still keep this definition since it is very convenient, as we shall see. To an order consistent with (2.1), we can express the previous "symmetric" potentials as

$$
\begin{aligned}
& V^{\mathrm{sym}}=U+\frac{1}{2 c^{2}} \partial_{t}^{2} X+\frac{1}{c^{4}} V_{4}^{\mathrm{sym}}+\frac{1}{c^{6}} V_{6}^{\mathrm{sym}}+\mathcal{O}\left(\frac{1}{c^{8}}\right), \\
& V_{i}^{\mathrm{sym}}=U_{i}+\frac{1}{c^{2}} V_{2}^{\mathrm{sym}}+\frac{1}{c^{4}} V_{4}^{\mathrm{sym}}+\mathcal{O}\left(\frac{1}{c^{6}}\right),
\end{aligned}
$$

where we do not need to control the $2 \mathrm{PN}$ and $3 \mathrm{PN}$ terms while we have introduced the usual Poisson integrals $U_{\mu}$ and the "super-potential" $X$ (only for the scalar potential) defined by:

$$
U_{\mu}=G \int \frac{\mathrm{d}^{3} \mathbf{x}^{\prime}}{\left|\mathbf{x}-\mathbf{x}^{\prime}\right|} \sigma_{\mu}\left(\mathbf{x}^{\prime}, t\right), \quad X=G \int \mathrm{d}^{3} \mathbf{x}^{\prime}\left|\mathbf{x}-\mathbf{x}^{\prime}\right| \sigma\left(\mathbf{x}^{\prime}, t\right) .
$$


As for the tensorial part $W_{i j}$ in the metric (2.1), it is given (modulo some constant factor) by the Poisson integral of the matter stresses $\sigma_{i j}$ plus the usual Newtonian gravitational stresses:

$$
W_{i j}=G \int \frac{\mathrm{d}^{3} \mathbf{x}^{\prime}}{\left|\mathbf{x}-\mathbf{x}^{\prime}\right|}\left[\sigma_{i j}+\frac{1}{4 \pi G}\left(\partial_{i} U \partial_{j} U-\frac{1}{2} \delta_{i j} \partial_{k} U \partial_{k} U\right)\right] .
$$

The radiation-reaction effects in $W_{i j}$ are negligible within the present approximation.

All the information regarding radiation reaction is "implicitly" contained in the matter currents $\sigma_{\mu}$ (as we have seen), and "explicitly" into the scalar and vector radiation-reaction potentials $V_{\mu}^{\text {reac }}=\left(V^{\text {reac }}, V_{i}^{\text {reac }}\right)$. Those are defined in terms of the multipole moments $I_{L}(t)$ and $J_{L}(t)$ of the post-Newtonian source as $[21,22]^{2}$

$$
\begin{aligned}
V^{\text {reac }} & =-\frac{G}{5 c^{5}} x_{i j} \stackrel{(5)}{I_{i j}}+\frac{G}{c^{7}}\left[\frac{1}{189} x_{i j k} \stackrel{(7)}{I_{i j k}}-\frac{1}{70} r^{2} x_{i j} \stackrel{(7)}{I_{i j}}\right]+\mathcal{O}\left(\frac{1}{c^{8}}\right), \\
V_{i}^{\text {reac }} & =\frac{G}{c^{5}}\left[\frac{1}{21} \hat{x}_{i j k} \stackrel{(6)}{I_{j k}}-\frac{4}{45} \varepsilon_{i j k} x_{j l} \stackrel{(5)}{J_{k l}}\right]+\mathcal{O}\left(\frac{1}{c^{7}}\right) .
\end{aligned}
$$

At the $4 \mathrm{PN}$ order the scalar potential $V^{\text {reac }}$ contains the contribution of tails [22], which is not needed here [but see Eq. (3.22) below]. Moreover, at the 4.5PN order, there should also be a genuine tensorial contribution to the radiation-reaction force. When restricted, by contrast, to the leading $2.5 \mathrm{PN}$ order, the potential $V^{\text {reac }}$ reduces to the Burke-Thorne radiation-reaction scalar potential $[5,6]$. The multipole moments are the mass quadrupole moment $I_{i j}$, consistently given here with $1 \mathrm{PN}$ accuracy [19],

$$
I_{i j}=\int \mathrm{d}^{3} \mathbf{x}\left[\hat{x}_{i j} \sigma+\frac{1}{14 c^{2}} r^{2} \hat{x}_{i j} \partial_{t}^{2} \sigma-\frac{20}{21 c^{2}} \hat{x}_{i j k} \partial_{t} \sigma_{k}\right]+\mathcal{O}\left(\frac{1}{c^{4}}\right),
$$

the mass octupole moment $I_{i j k}$ and the current quadrupole $J_{i j}$, which are merely Newtonian:

$$
I_{i j k}=\int \mathrm{d}^{3} \mathbf{x} \hat{x}_{i j k} \sigma+\mathcal{O}\left(\frac{1}{c^{2}}\right), \quad J_{i j}=\int \mathrm{d}^{3} \mathbf{x} \varepsilon_{k l(i} x_{j) k} \sigma_{l}+\mathcal{O}\left(\frac{1}{c^{2}}\right)
$$

\section{FLUX-BALANCE EQUATIONS TO 3.5PN ORDER}

The metric (2.1) is used in the derivation of the flux-balance equations for linear momentum and (in an extension of [22]) for the position of the center of mass; concerning the energy and angular momentum, we shall simply restate the results of [22]. The method consists of integrating the equations of motion over a volume enclosing the compact-support matter

\footnotetext{
${ }^{2}$ The multipole moments are symmetric and trace-free (STF) with respect to their $\ell$ indices $L=i_{1} i_{2} \cdots i_{\ell}$; we denote the STF product of spatial vectors $x_{i}$ as $\hat{x}_{L}=\operatorname{STF}\left(x_{L}\right)$, so that $\hat{x}_{i j}=x_{i j}-\frac{1}{3} \delta_{i j} r^{2}, \hat{x}_{i j k}=$ $x_{i j k}-\frac{1}{5}\left(\delta_{i j} x_{k}+\delta_{i k} x_{j}+\delta_{j k} x_{i}\right) r^{2}$ and so on (with $r=|\mathbf{x}|, x_{i j}=x_{i} x_{j}$ and $x_{i j k}=x_{i} x_{j} x_{k}$ ); similarly, we denote $\partial_{L}=\partial_{i_{1}} \partial_{i_{1}} \cdots \partial_{i_{\ell}}$ and $\hat{\partial}_{L}=\operatorname{STF}\left(\partial_{L}\right) ; \varepsilon_{i j k}$ is the fully antisymmetric Levi-Cevita symbol. Notice that all products of spatial vectors in Eqs. (2.8) are actually STF. On the other hand, parenthesis surrounding indices mean their symmetrization: $t_{(i j)}=\frac{1}{2}\left(t_{i j}+t_{j i}\right)$ whereas superscripts $(n)$ stand for $n$ time derivatives.
} 
distribution of the source, the equations of motion being here the covariant conservation of the matter stress-energy tensor, $\nabla_{\nu} T_{\mu}^{\nu}=0$, rewritten in the more convenient way as

$$
\partial_{\nu} \Pi_{\mu}^{\nu}=\mathcal{F}_{\mu}
$$

where we pose $\Pi_{\mu}^{\nu}=\sqrt{-g} T_{\mu}^{\nu}$ and $\mathcal{F}_{\mu}=\frac{1}{2} \sqrt{-g} \partial_{\mu} g_{\rho \sigma} T^{\rho \sigma}$, with $T_{\mu}^{\nu}=g_{\mu \rho} T^{\nu \rho}$ and $g=\operatorname{det}\left(g_{\rho \sigma}\right)$. By substituting (2.1) and using the definitions of the mass, current and stress densities (2.2), we obtain explicit expressions containing all radiation effects up to 3.5PN order but in which the conservative effects at $2 \mathrm{PN}$ and $3 \mathrm{PN}$ orders are neglected. The components of the effective force in the RHS read

$$
\begin{aligned}
& \mathcal{F}_{0}=\frac{1}{c} \sigma \partial_{t} \mathcal{V}-\frac{4}{c^{3}} \sigma_{j} \partial_{t} \mathcal{V}_{j}+\frac{1}{c^{5}}{\underset{F}{5}}_{0}+\frac{1}{c^{7}} \mathcal{F}_{7}+\mathcal{O}\left(\frac{1}{c^{9}}\right), \\
& \mathcal{F}_{i}=\sigma \partial_{i} \mathcal{V}-\frac{4}{c^{2}} \sigma_{j} \partial_{i} \mathcal{V}_{j}+\frac{1}{c^{4}}{\underset{F}{i}}_{i}+\frac{1}{c^{6}} \underset{6}{\mathcal{F}_{i}}+\mathcal{O}\left(\frac{1}{c^{8}}\right),
\end{aligned}
$$

while those of the effective momentum in the LHS are

$$
\begin{aligned}
& \Pi_{0}^{0}=-c^{2} \sigma+\sigma_{i i}+\frac{4}{c^{2}}\left(\sigma W_{i i}-\sigma_{i} \mathcal{V}_{i}\right)+\frac{1}{c^{4}} \Pi_{4}^{0}+\frac{1}{c^{6}} \Pi_{6}^{0}+\mathcal{O}\left(\frac{1}{c^{8}}\right), \\
& \Pi_{i}^{0}=c \sigma_{i}+\frac{4}{c}\left(\sigma_{i} \mathcal{V}-\sigma \mathcal{V}_{i}\right)+\frac{1}{c^{3}} \Pi_{3}^{0}+\frac{1}{c^{5}} \Pi_{5}^{0}+\mathcal{O}\left(\frac{1}{c^{7}}\right), \\
& \Pi_{0}^{i}=-c \sigma_{i}+\frac{4}{c^{3}}\left(\sigma_{i} W_{j j}-\sigma_{i j} \mathcal{V}_{j}\right)+\frac{1}{c^{5}} \prod_{5}^{i}+\mathcal{O}\left(\frac{1}{c^{7}}\right), \\
& \Pi_{j}^{i}=\sigma_{i j}+\frac{4}{c^{2}}\left(\sigma_{i j} \mathcal{V}-\sigma_{i} \mathcal{V}_{j}\right)+\frac{1}{c^{4}} \prod_{4}^{i}+\frac{1}{c^{6}} \Pi_{6}^{i}+\mathcal{O}\left(\frac{1}{c^{8}}\right) .
\end{aligned}
$$

The $1 \mathrm{PN}$ equation of continuity and the $1 \mathrm{PN}$ equation of motion (or relativistic Euler equation) are explicitly given in the present formalism by

$$
\begin{aligned}
& \partial_{t} \sigma+\partial_{i} \sigma_{i}=\frac{1}{c^{2}}\left(\partial_{t} \sigma_{j j}-\sigma \partial_{t} \mathcal{V}\right)+\overline{\mathcal{O}}\left(\frac{1}{c^{8}}\right) \\
& \partial_{t}\left[\sigma_{i}\left(1+\frac{4 \mathcal{V}}{c^{2}}\right)\right]+\partial_{j}\left[\sigma_{i j}\left(1+\frac{4 \mathcal{V}}{c^{2}}\right)\right]=\sigma \partial_{i} \mathcal{V}+\frac{4}{c^{2}}\left[\sigma \partial_{t} \mathcal{V}_{i}+\sigma_{j}\left(\partial_{j} \mathcal{V}_{i}-\partial_{i} \mathcal{V}_{j}\right)\right]+\overline{\mathcal{O}}\left(\frac{1}{c^{8}}\right)
\end{aligned}
$$

The special notation for the remainder term means that it is correct regarding radiationreaction effects up to $3.5 \mathrm{PN}$ order included, but contains some uncontrolled $2 \mathrm{PN}$ and $3 \mathrm{PN}$ conservative contributions. Namely, the remainders in (3.4) are of the type

$$
\overline{\mathcal{O}}\left(\frac{1}{c^{8}}\right)=\frac{1}{c^{4}} \underset{4}{X}+\frac{1}{c^{6}} \underset{6}{X}+\mathcal{O}\left(\frac{1}{c^{8}}\right) .
$$

The radiation-reaction parts (2.8) of the potentials $\mathcal{V}_{\mu}$ appear explicitly through the dependence of the laws (3.4) upon $\mathcal{V}_{\mu}$, but also implicitly through the matter currents and stresses, $\sigma_{\mu}$ and $\sigma_{i j}$, respectively. We find that only the former will contribute to the fluxes in the RHS of the balance equations, while the latter implicit radiation-reaction terms appear only as total time derivatives in the LHS. In order to obtain the radiation-reaction contributions 
in the stress-energy tensor $T^{\mu \nu}$, one must identify the matter degrees of freedom, independently from the gravitational field (i.e., the metric). For a perfect fluid system, the matter degrees of freedom can be chosen to be the coordinate-velocity field $v^{\mu}=c u^{\mu} / u^{0}$, the specific entropy $s_{*}$, and the coordinate density $\rho_{*}=\sqrt{-g} \rho u^{0}$, where $\rho$ is the conserved scalar density satisfying $\nabla_{\mu}\left(\rho u^{\mu}\right)=0$, and $u^{0}=\left(-g_{\mu \nu} v^{\mu} v^{\nu} / c^{2}\right)^{-1 / 2}$. In this way, we find for instance $\sigma^{\text {reac }}=\rho_{*} V^{\text {reac }} / c^{2}+\mathcal{O}\left(1 / c^{9}\right)$, which is a $3.5 \mathrm{PN}$ effect.

\section{A. Linear momentum}

The balance equation for linear momentum has already been derived in [22], but we repeat here the main steps for completeness. We first integrate Eq. (3.1) with $\mu=i$ over the compact-support matter distribution. Using the Gauss law to discard a total divergence, as well as the expressions (3.2b) and (3.3b), we thus obtain

$$
\frac{\mathrm{d}}{\mathrm{d} t}\left(\int \mathrm{d}^{3} \mathbf{x}\left[\sigma_{i}+\frac{4}{c^{2}}\left(\sigma_{i} \mathcal{V}-\sigma \mathcal{V}_{i}\right)\right]\right)=\int \mathrm{d}^{3} \mathbf{x}\left(\sigma \partial_{i} \mathcal{V}-\frac{4}{c^{2}} \sigma_{j} \partial_{i} \mathcal{V}_{j}\right)+\overline{\mathcal{O}}\left(\frac{1}{c^{8}}\right)
$$

We then split the potentials $\mathcal{V}_{\mu}$ into symmetric and reaction parts [see Eq. (2.3)] and perform simple manipulations. In particular, we use a generalization of the "action-reaction" theorem valid at the $1 \mathrm{PN}$ order, namely

$$
\int \mathrm{d}^{3} \mathbf{x} \sigma \partial_{i} V^{\mathrm{sym}}=\frac{\mathrm{d}}{\mathrm{d} t}\left[\frac{1}{2 c^{2}} \int \mathrm{d}^{3} \mathbf{x} \sigma \partial_{i} \partial_{t} X\right]+\mathcal{O}\left(\frac{1}{c^{4}}\right),
$$

where $X$ is the super-potential defined in (2.6). This permits transfering a term in the form of a total time derivative to the LHS of the equation, yielding

$$
\begin{gathered}
\frac{\mathrm{d}}{\mathrm{d} t}\left(\int \mathrm{d}^{3} \mathbf{x}\left[\sigma_{i}-\frac{1}{2 c^{2}} \sigma \partial_{i} \partial_{t} X+\frac{4}{c^{2}}\left(\sigma_{i} V^{\text {reac }}-\sigma V_{i}^{\text {reac }}\right)\right]+\overline{\mathcal{O}}\left(\frac{1}{c^{8}}\right)\right) \\
=\int \mathrm{d}^{3} \mathbf{x}\left(\sigma \partial_{i} V^{\text {reac }}-\frac{4}{c^{2}} \sigma_{j} \partial_{i} V_{j}^{\text {reac }}\right)+\mathcal{O}\left(\frac{1}{c^{9}}\right) .
\end{gathered}
$$

In this equation, we have also transferred the uncontrolled $2 \mathrm{PN}$ and $3 \mathrm{PN}$ approximations from the RHS to the LHS, where they now lie inside the remainder [see our special notation (3.5)]. Indeed, these approximations are conservative so that they must appear in the form of total time derivatives in our flux-balance equations. This has been proven long ago for general fluids at the $2 \mathrm{PN}$ approximation [7, 8], but we assume here that the same is true for the $3 \mathrm{PN}$ terms, for the conservative terms at the 4PN approximation (i.e., apart for the tail term), and so on. This assumption has been fully confirmed by recent works on the equations of motion of compact binary systems at the 4PN order, which showed the existence at that order of all the Poincaré invariants (see notably [62], and Sec. V below).

At this stage, the radiation-reaction potentials appear on both sides of Eq. (3.8). In the next step, we replace the reaction potentials in the RHS by their explicit expressions provided by Eqs. (2.8). A straightforward computation leads then to the usual flux-balance equation for linear momentum at the leading 3.5PN order:

$$
\frac{\mathrm{d} P_{i}}{\mathrm{~d} t}=-\frac{G}{c^{7}}\left[\frac{2}{63} \stackrel{(4)}{I}_{i j k} \stackrel{(3)}{I_{j k}}+\frac{16}{45} \varepsilon_{i j k} \stackrel{(3)}{I_{j l}} \stackrel{(3)}{J}_{k l}\right]+\mathcal{O}\left(\frac{1}{c^{9}}\right)
$$


where, importantly for the precise meaning of the equation, the linear momentum $P_{i}$ in the LHS is explicitly given by

$$
P_{i}=\int \mathrm{d}^{3} \mathbf{x}\left[\sigma_{i}-\frac{1}{2 c^{2}} \sigma \partial_{i} \partial_{t} X+\frac{4}{c^{2}}\left(\sigma_{i} V^{\text {reac }}-\sigma V_{i}^{\text {reac }}\right)\right]+\delta P_{i}^{\text {reac }}+\overline{\mathcal{O}}\left(\frac{1}{c^{8}}\right) .
$$

Besides the neglected conservative $2 \mathrm{PN}$ and $3 \mathrm{PN}$ terms included in the remainder, there appears a term $\delta P_{i}^{\text {reac }}$, composed of $2.5 \mathrm{PN}$ and $3.5 \mathrm{PN}$ approximations, which comes from the total time derivatives originally present in the RHS. As it will play a major role in what follows, we display it explicitly:

$$
\begin{aligned}
& \delta P_{i}^{\mathrm{reac}}=\frac{2 G}{5 c^{5}}\left(I_{j} \stackrel{(4)}{I}_{i j}-\stackrel{(1)}{I_{j}} \stackrel{(3)}{I}_{i j}\right) \\
& +\frac{G}{c^{7}}\left[\frac{8}{15} J_{j} J_{i j}^{(4)}-\frac{1}{63} \stackrel{(6)}{I}_{i j k} I_{j k}+\frac{1}{63} \stackrel{(5)}{I}_{i j k} \stackrel{(1)}{I_{j k}}-\frac{1}{63} \stackrel{(4)}{I}_{i j k} \stackrel{(2)}{I}_{j k}-\frac{1}{63} \stackrel{(3)}{I}_{i j k} \stackrel{(3)}{I}_{j k}\right. \\
& +\frac{1}{63} \stackrel{(2)}{I}_{i j k} \stackrel{(4)}{I}_{j k}-\frac{1}{63} \stackrel{(1)}{I}_{i j k} \stackrel{(5)}{I}_{j k}+\frac{1}{35} I_{i j k} \stackrel{(6)}{I}_{j k} \\
& -\frac{8}{45} \varepsilon_{i j k} \stackrel{(4)}{J_{j l}} \stackrel{(1)}{I}_{k l}+\frac{8}{45} \varepsilon_{i j k} \stackrel{(3)}{J_{j l}} \stackrel{(2)}{I}_{k l}+\frac{8}{45} \varepsilon_{i j k} \stackrel{(2)}{J_{j l}} \stackrel{(3)}{I}_{k l}-\frac{8}{45} \varepsilon_{i j k} \stackrel{(1)}{J_{j l}} \stackrel{(4)}{I}_{k l} \\
& \left.+\frac{8}{45} \varepsilon_{i j k} J_{j l} \stackrel{(5)}{I_{k l}}+\frac{2}{5} K_{j} \stackrel{(5)}{I}_{i j}-\frac{1}{5} L_{j} \stackrel{(5)}{I}_{i j}+\frac{1}{25} N_{j} \stackrel{(6)}{I}_{i j}\right] .
\end{aligned}
$$

The mass quadrupole moment $I_{i j}$ already enters this expression at the 2.5PN order and is thus consistently given with $1 \mathrm{PN}$ accuracy by Eq. (2.9). In addition, the leading terms depend on the mass dipole moment, which is required with the same 1PN accuracy and reads

$$
I_{i}=\int \mathrm{d}^{3} \mathbf{x} x_{i}\left(\sigma+\frac{1}{c^{2}}\left[\frac{\sigma U}{2}-\sigma_{j j}\right]\right)+\mathcal{O}\left(\frac{1}{c^{4}}\right) .
$$

For the expressions of the other moments in (3.11), which are simply Newtonian, one should refer to (2.10). Furthermore, $\delta P_{i}^{\text {reac }}$ contains the conserved Newtonian angular momentum of the system identified with the current-type dipole moment,

$$
J_{i}=\int \mathrm{d}^{3} \mathbf{x} \varepsilon_{i j k} x_{j} \sigma_{k}+\mathcal{O}\left(\frac{1}{c^{2}}\right),
$$

such that $\mathrm{d} J_{i} / \mathrm{d} t=\mathcal{O}\left(1 / c^{2}\right)$, as well as three suplementary integrals defined at Newtonian order only,

$$
K_{i}=\int \mathrm{d}^{3} \mathbf{x} \sigma_{j} x_{i j}, \quad L_{i}=\int \mathrm{d}^{3} \mathbf{x} \sigma_{i} r^{2}, \quad N_{i}=\int \mathrm{d}^{3} \mathbf{x} \sigma x_{i} r^{2}
$$

These integrals are not independent but are linked together, as a consequence of the continuity equation (3.4a), by $N_{i}^{(1)}=2 K_{i}+L_{i}+\mathcal{O}\left(1 / c^{2}\right)$.

\section{B. Center-of-mass position}

We now want to rewrite the linear momentum $P_{i}$ defined by Eqs. (3.10)-(3.11) as a total time derivative plus radiation-reaction terms. The time derivative will be naturally 
interpreted as that of the position of the center of mass $G_{i}$ of the matter system (multiplied by its mass) which, for gravitating systems, is nothing but the mass dipole moment $I_{i}$, so that we shall recover Eq. (3.12) in the $1 \mathrm{PN}$ approximation. Multiplying the continuity equation (3.4a) by $x_{i}$ and integrating we get

$$
\frac{\mathrm{d}}{\mathrm{d} t}\left[\int \mathrm{d}^{3} \mathbf{x} x_{i}\left(\sigma-\frac{1}{c^{2}} \sigma_{j j}\right)\right]=\int \mathrm{d}^{3} \mathbf{x}\left[\sigma_{i}-\frac{1}{c^{2}} x_{i} \sigma \partial_{t} \mathcal{V}\right]+\overline{\mathcal{O}}\left(\frac{1}{c^{8}}\right) .
$$

Thanks to that relation, we can transform the expression (3.10) as

$$
\begin{aligned}
P_{i}=\frac{\mathrm{d}}{\mathrm{d} t}\left[\int\right. & \left.\mathrm{d}^{3} \mathbf{x} x_{i}\left(\sigma+\frac{1}{c^{2}}\left[\frac{\sigma U}{2}-\sigma_{j j}\right]\right)\right] \\
& +\frac{1}{c^{2}} \int \mathrm{d}^{3} \mathbf{x}\left[x_{i} \sigma \partial_{t} V^{\text {reac }}+4\left(\sigma_{i} V^{\text {reac }}-\sigma V_{i}^{\text {reac }}\right)\right]+\delta P_{i}^{\text {reac }}+\overline{\mathcal{O}}\left(\frac{1}{c^{8}}\right) .
\end{aligned}
$$

Next, inserting the values (2.3) of the radiation-reaction potentials $V_{\mu}^{\text {reac }}$ and combining the result with the expression (3.11), we observe important simplifications, modulo total time derivatives. Notably, the contributions from the current quadrupole moment, as well as those from the extra integrals (3.14), can all be absorbed into a total time derivative which will constitute an analogue of the Schott terms in the flux-balance equation for the centerof-mass position. Finally, we find that Eqs. (3.10)-(3.11) can equivalently be rewritten as

$$
\frac{\mathrm{d} G_{i}}{\mathrm{~d} t}=P_{i}-\frac{2 G}{21 c^{7}} \stackrel{(3)}{I}_{i j k} \stackrel{(3)}{I}_{j k}+\mathcal{O}\left(\frac{1}{c^{9}}\right)
$$

where $G_{i}$ is given by

$$
G_{i}=\int \mathrm{d}^{3} \mathbf{x} x_{i}\left(\sigma+\frac{1}{c^{2}}\left[\frac{\sigma U}{2}-\sigma_{j j}\right]\right)+\delta G_{i}^{\mathrm{reac}}+\overline{\mathcal{O}}\left(\frac{1}{c^{8}}\right) .
$$

We recover, as expected, the expression of the mass-type dipole moment (3.12). The radiation-reaction terms $\delta G_{i}^{\text {reac }}$ are given by

$$
\begin{aligned}
& \delta G_{i}^{\mathrm{reac}}=\frac{2 G}{5 c^{5}}\left(I_{j} \stackrel{(3)}{I}_{i j}-2 \stackrel{(1)}{I_{j}} \stackrel{(2)}{I}_{i j}\right) \\
& +\frac{G}{c^{7}}\left[-\frac{1}{63} \stackrel{(5)}{I}_{i j k} I_{j k}+\frac{2}{63} \stackrel{(4)}{I}_{i j k} \stackrel{(1)}{I}_{j k}-\frac{1}{21} \stackrel{(3)}{I}_{i j k} \stackrel{(2)}{I}_{j k}+\frac{19}{315} \stackrel{(2)}{I}_{i j k} \stackrel{(3)}{I}_{j k}-\frac{2}{45} \stackrel{(1)}{I}_{i j k} \stackrel{(4)}{I_{j k}}+\frac{1}{35} I_{i j k} \stackrel{(5)}{I_{j k}}\right. \\
& -\frac{8}{45} \varepsilon_{i j k} \stackrel{(3)}{J_{j l}} \stackrel{(1)}{I}_{k l}+\frac{8}{15} \varepsilon_{i j k} \stackrel{(2)}{J_{j l}} \stackrel{(2)}{I}_{k l}-\frac{16}{45} \varepsilon_{i j k} \stackrel{(1)}{J_{j l}} \stackrel{(3)}{I}_{k l}+\frac{8}{45} \varepsilon_{i j k} J_{j l} \stackrel{(4)}{I}_{k l} \\
& \left.+\frac{1}{25} N_{j} \stackrel{(5)}{I_{i j}}+\frac{8}{15} J_{j} \stackrel{(3)}{J}_{i j}\right] \text {. }
\end{aligned}
$$

As usual, the latter terms (3.19), although of the same PN order as the flux terms in the RHS of (3.17), will in fact be very small in the adiabatic approximation, i.e., when considered in average over a typical oscillation period of the system. Finally, as we discussed in the introduction, the result (3.17) forms an integral part of the description of the secular evolution of an isolated system due to gravitational radiation. 


\section{Energy and angular momentum}

For completeness, we also present the balance equations obeyed by the energy and angular momentum, following [22]. With the precision of the metric (2.1), we are able to write the balance equations at the subleading $1 \mathrm{PN}$ order for conservative effects and subleading 3.5PN order for radiation-reaction effects. These $1 \mathrm{PN}$ relative equations involve the well known $1 \mathrm{PN}$ fluxes in the RHS,

$$
\begin{aligned}
\frac{\mathrm{d} E}{\mathrm{~d} t} & =-\frac{G}{c^{5}}\left(\frac{1}{5} \stackrel{(3)}{I}_{i j} \stackrel{(3)}{I}_{i j}+\frac{1}{c^{2}}\left[\frac{1}{189} \stackrel{(4)}{I}_{i j k} \stackrel{(4)}{I}_{i j k}+\frac{16}{45} \stackrel{(3)}{J}_{i j} \stackrel{(3)}{J_{i j}}\right]\right)+\mathcal{O}\left(\frac{1}{c^{8}}\right), \\
\frac{\mathrm{d} J_{i}}{\mathrm{~d} t} & =-\frac{G}{c^{5}} \varepsilon_{i j k}\left(\frac{2}{5} \stackrel{(2)}{I}_{j l} \stackrel{(3)}{I}_{k l}+\frac{1}{c^{2}}\left[\frac{1}{63} \stackrel{(3)}{I}_{j l m} \stackrel{(4)}{I}_{k l m}+\frac{32}{45} \stackrel{(2)}{J}_{j l}^{(3)} \stackrel{(3)}{k l}_{k l}\right]\right)+\mathcal{O}\left(\frac{1}{c^{8}}\right),
\end{aligned}
$$

where the $1 \mathrm{PN}$ mass quadrupole moment $I_{i j}$ is given by (2.9). The quantities in the LHS, with consistent accuracy, read

$$
\begin{aligned}
E & =\int \mathrm{d}^{3} \mathbf{x}\left(\sigma c^{2}+\frac{1}{2} \sigma U-\sigma_{i i}+\frac{1}{c^{2}}\left[-4 \sigma W_{i i}+2 \sigma_{i} U_{i}+\frac{1}{2} \sigma \partial_{t}^{2} X-\frac{1}{4} \partial_{t} \sigma \partial_{t} X\right]\right) \\
& +\delta E^{\mathrm{reac}}+\overline{\mathcal{O}}\left(\frac{1}{c^{8}}\right), \\
J_{i} & =\varepsilon_{i j k} \int \mathrm{d}^{3} \mathbf{x} x_{j}\left(\sigma_{k}+\frac{1}{c^{2}}\left[4 \sigma_{k} U-4 \sigma U_{k}-\frac{1}{2} \sigma \partial_{k} \partial_{t} X\right]\right)+\delta J_{i}^{\text {reac }}+\overline{\mathcal{O}}\left(\frac{1}{c^{8}}\right),
\end{aligned}
$$

where $\delta E^{\text {reac }}$ and $\delta J_{i}^{\text {reac }}$ represent some $2.5 \mathrm{PN}$ and $3.5 \mathrm{PN}$ contributions coming from total time derivatives appearing in the RHS, and which will not be needed here. Notice that, because of the scaling of the rest mass contribution $\sigma c^{2}$ in the energy, the relative precision of the expression (3.21a) is actually $2 \mathrm{PN}$, i.e., the total mass $M=E / c^{2}$ is given at the $2 \mathrm{PN}$ order. This is why we had to push the accuracy of the spatial part $g_{i j}$ of the metric (2.1) up to $2 \mathrm{PN}$ order and include the terms involving the potential $W_{i j}$ defined by Eq. (2.7).

Finally, we recall from [22] that we can even include the tail effect at the 4PN order, since the radiation-reaction potential $V^{\text {reac }}$ contains a tail contribution at that order. The $4 \mathrm{PN}$ energy balance equation, for instance, becomes

$$
\begin{aligned}
\frac{\mathrm{d} E}{\mathrm{~d} t}= & -\frac{G}{5 c^{5}}\left(\stackrel{(3)}{I_{i j}}(t)+\frac{G M}{c^{3}} \int_{0}^{+\infty} \mathrm{d} \tau \ln \tau\left[\stackrel{(5)}{I_{i j}}(t-\tau)+\stackrel{(5)}{I_{i j}}(t+\tau)\right]\right)^{2} \\
& -\frac{G}{c^{7}}\left[\frac{1}{189}\left(\stackrel{(4)}{I_{i j k}}\right)^{2}+\frac{16}{45}\left(\stackrel{(3)}{J}_{i j}\right)^{2}\right]+\mathcal{O}\left(\frac{1}{c^{9}}\right)
\end{aligned}
$$

Note that the tail term in the RHS is given here as an "antisymmetric" integral, which corresponds in fact to the dissipative part of the full tail effect. ${ }^{3}$ The energy in the LHS of Eq. (3.22) also acquires some 4PN tail induced contributions but in the form of some "symmetric" integrals, which have been investigated, e.g., in Sec. IV of [62].

\footnotetext{
${ }^{3}$ Any constant inserted into the logarithmic kernel of that antisymmetric integral cancels out.
} 


\section{DIRECT COMPUTATIONS OF THE GRAVITATIONAL-WAVE FLUXES}

In this section, we perform a completely different type of calculation. Namely, we compute directly the fluxes appearing in the RHS of the balance equations from the matter source at future null infinity $\mathscr{I}^{+}$. This will permit to check our new balance equation (3.17) for the position of the center of mass. In fact, we shall obtain the full multipole moment expansion for the flux, going beyond the leading order. We shall also recover the known multipolar expansions for the fluxes associated with the other invariants. Our calculation, however, will be restricted to quadratic order in a post-Minkowskian expansion $\left(G^{2}\right)$.

\section{A. Integral conservation identities}

We start with the gauge-fixed Einstein field equations in harmonic coordinates,

$$
\square h^{\mu \nu}=\frac{16 \pi G}{c^{4}} \tau^{\mu \nu}
$$

where $\square$ is the flat d'Alembertian operator. In addition, the field variable $h^{\mu \nu}=\sqrt{-g} g^{\mu \nu}-$ $\eta^{\mu \nu}$ (i.e. the "gothic" metric deviation from Minkowski's metric) must satisfy the harmonic gauge condition $\partial_{\nu} h^{\mu \nu}=0$. The pseudo stress-energy tensor in the RHS,

$$
\tau^{\mu \nu}=|g| T^{\mu \nu}+\frac{c^{4}}{16 \pi G} \Lambda^{\mu \nu}
$$

is the sum of the matter contribution with compact support and the non-linear gravitational source term $\Lambda^{\mu \nu}$, which is at least quadratic in $h^{\mu \nu}$ and its space-time derivatives.

To derive the flux-balance equations, we integrate the conservation law $\partial_{\nu} \tau^{\mu \nu}=0$ (or similar relations following from it) over a three-dimensional volume $\mathscr{V}$ enclosing the compactsupport source and bounded by some two-dimensional surface $\mathscr{S}$. Since we look for the balance equations describing the evolution, due to gravitational-wave emission, of otherwise constant quantities, the volume $\mathscr{V}$ is chosen to tend asymptotically toward $\mathscr{I}^{+}$. It is thus natural to perform a change of coordinates $(t, \mathbf{x}) \longrightarrow(u, \mathbf{x})$ where $u$ denotes an outgoing null coordinate, satisfying $g^{\mu \nu} \partial_{\mu} u \partial_{\nu} u=0$. For simplicity, we take it to be of the form $u=t-r_{*}(\mathbf{x}) / c$, where the "tortoise" coordinate $r_{*}$ depends on position $\mathbf{x}$ but not on time $t$. At leading order, the tortoise coordinate contains the well known logarithmic deviation of light cones in harmonic coordinates, $r_{*}=r+\frac{2 G M}{c^{2}} \ln r+\mathcal{O}\left(r^{0}\right)$. Posing $n_{*}^{i}=\partial_{i} r_{*}$ we can rewrite the conservation law of the pseudo tensor $\tau^{\mu \nu}$ in the coordinate system $(u, \mathbf{x}) \operatorname{as}^{4}$

$$
\frac{\partial}{c \partial u}\left[\tau^{\mu 0}\left(\mathbf{x}, u+r_{*} / c\right)-n_{*}^{i} \tau^{\mu i}\left(\mathbf{x}, u+r_{*} / c\right)\right]+\partial_{i}\left[\tau^{\mu i}\left(\mathbf{x}, u+r_{*} / c\right)\right]=0 .
$$

In this approach, all volume integrals are defined over the finite volume $\mathscr{V}$, while the surface integrals produced when applying Gauss's law and used to construct the fluxes are computed over the two-dimensional boundary $\mathscr{S}=\partial \mathscr{V}$ of that volume. In the end, we shall formally let the volume grow to infinity and the surface $\mathscr{S}$ tend toward $\mathscr{I}^{+}$, in the limit

${ }^{4}$ The nullity condition of the $u$-coordinate, $g^{\mu \nu} \partial_{\mu} u \partial_{\nu} u=0$, translates into a condition on the Euclidean norm of the vector $n_{*}^{i}$, namely $\boldsymbol{n}_{*}^{2}=1-h^{00}+2 h^{0 i} n_{*}^{i}-h^{i j} n_{*}^{i} n_{*}^{j}$. 
$r \rightarrow+\infty$ at $u=$ const. It turns out that all the fluxes are convergent in this limit modulo total time derivatives. Following this procedure, it is straightforward to obtain the following flux-balance equations ${ }^{5}$

$$
\begin{aligned}
\frac{\mathrm{d} E}{\mathrm{~d} u} & =-c \oint_{\mathscr{S}} \mathrm{d} S_{i} \tau_{\mathrm{GW}}^{0 i}\left(\mathbf{x}, u+r_{*} / c\right), \\
\frac{\mathrm{d} P^{i}}{\mathrm{~d} u} & =-\oint_{\mathscr{S}} \mathrm{d} S_{j} \tau_{\mathrm{GW}}^{i j}\left(\mathbf{x}, u+r_{*} / c\right), \\
\frac{\mathrm{d} J_{i}}{\mathrm{~d} u} & =-\varepsilon_{i j k} \oint_{\mathscr{S}} \mathrm{d} S_{l} x^{j} \tau_{\mathrm{GW}}^{k l}\left(\mathbf{x}, u+r_{*} / c\right), \\
\frac{\mathrm{d} G_{i}}{\mathrm{~d} u} & =P_{i}-\frac{1}{c} \oint_{\mathscr{S}} \mathrm{d} S_{j}\left(x^{i} \tau_{\mathrm{GW}}^{0 j}-r_{*} \tau_{\mathrm{GW}}^{i j}\right)\left(\mathbf{x}, u+r_{*} / c\right),
\end{aligned}
$$

where we have denoted quite naturally $\tau_{\mathrm{GW}}^{\mu \nu}=\frac{c^{4}}{16 \pi G} \Lambda^{\mu \nu}$. The quantities on the LHS are the Bondi-like energy, linear momentum, angular momentum (or current-type dipole moment) and center-of-mass position (or mass-type dipole moment):

$$
\begin{aligned}
E & =\int_{\mathscr{V}} \mathrm{d}^{3} \mathbf{x}\left[\tau^{00}-n_{*}^{i} \tau^{0 i}\right]\left(\mathbf{x}, u+r_{*} / c\right), \\
P^{i} & =\frac{1}{c} \int_{\mathscr{V}} \mathrm{d}^{3} \mathbf{x}\left[\tau^{i 0}-n_{*}^{j} \tau^{i j}\right]\left(\mathbf{x}, u+r_{*} / c\right), \\
J_{i} & =\frac{1}{c} \varepsilon_{i j k} \int_{\mathscr{V}} \mathrm{d}^{3} \mathbf{x} x^{j}\left[\tau^{k 0}-n_{*}^{l} \tau^{k l}\right]\left(\mathbf{x}, u+r_{*} / c\right), \\
G_{i} & =\frac{1}{c^{2}} \int_{\mathscr{V}} \mathrm{d}^{3} \mathbf{x}\left[x^{i}\left(\tau^{00}-n_{*}^{j} \tau^{0 j}\right)-r_{*}\left(\tau^{i 0}-n_{*}^{j} \tau^{i j}\right)\right]\left(\mathbf{x}, u+r_{*} / c\right) .
\end{aligned}
$$

\section{B. Multipolar expansion of the fluxes}

Next, we compute the fluxes in the RHS of Eqs. (4.4). The usual way to proceed consists of expanding the Einstein field equations when $r \rightarrow+\infty$ near $\mathscr{I}^{+}{ }^{6}$ This calculation is then "exact", as it requires only the leading and subleading expansion coefficients $1 / r^{2}$ and $1 / r^{3}$ of the GW stress-energy pseudo tensor in an appropriate radiative-type coordinate system. However, the fluxes are then given in terms of the radiative multipole moments (say $U_{L}$ and $V_{L}$ ), which represent a mere parametrization of the asymptotic waveform, disconnected from the matter source at this stage.

Here we adopt a different approach. Namely, we restrict the computation of the fluxes to dominant order in a post-Minkowskian expansion $(G \rightarrow 0)$, which amounts to considering only the quadratic non-linearities into the GW stress-energy pseudo tensor. The advantage is that the fluxes are now given in terms of some source-rooted multipole moments, say $M_{L}$

\footnotetext{
${ }^{5}$ See standard textbooks such as [11] and [54] for the derivation of the flux-balance equations. Note however that in the book [54] the integration is performed over a volume at constant time $t$ instead of constant outgoing null coordinate $u$, so that the computed quantities tend towards the ADM rather than Bondi values when the surface $\mathscr{S}$ goes to infinity. Note also that the flux integral for the center of mass we find in Eq. (4.4d) differs from Eq. (6.35) in Ref. [54].

${ }^{6}$ See most references in the field, from historical works, e.g., [47] [48] [29], till recent contributions, e.g., [63].
} 
and $S_{L}$, which are well controlled since they admit closed form expressions as integrals over the source, i.e., over the components of the pseudo tensor [23]. In the end, the multipole moment expansions we get should have the same structure as the "exact" fluxes written in terms of the radiative moments $U_{L}$ and $V_{L}$. Higher order effects such as tails can also be incorporated, with some more work, going to next order in $G$.

Let us notice that, to dominant order in $G$, we do not need to consider the logarithmic deviation of retarded null cones in harmonic coordinates, so that we can approximate $r_{*}$ by $r$ and $n_{*}^{i}$ by $n^{i}$. The GW pseudo tensor then reads at quadratic order

$$
\tau_{\mathrm{GW}}^{\mu \nu}=\frac{G c^{4}}{16 \pi} \Lambda_{2}^{\mu \nu}\left[h_{1}, h_{1}\right]+\mathcal{O}\left(G^{2}\right),
$$

where $\Lambda_{2}^{\mu \nu}$ is the quadratic piece of the non-linear source term in Eq. (4.2), with schematic form $\Lambda_{2}[h, h] \sim h \partial^{2} h+\partial h \partial h$. It is obtained by inserting the expression of the linearized metric in harmonic coordinates, $G h_{1}^{\mu \nu}$, given outside the matter source in the form of a full multipole moment expansion, through which the multipole moments of the source are precisely defined. This linearized multipolar solution of the vacuum field equations reads [29]

$$
\begin{aligned}
h_{1}^{00} & =-\frac{4}{c^{2}} \sum_{\ell=0}^{+\infty} \frac{(-)^{\ell}}{\ell !} \partial_{L}\left(\frac{1}{r} M_{L}(u)\right), \\
h_{1}^{0 i} & =\frac{4}{c^{3}} \sum_{\ell=1}^{+\infty} \frac{(-)^{\ell}}{\ell !}\left\{\partial_{L-1}\left(\frac{1}{r} \stackrel{(1)}{M}_{i L-1}(u)\right)+\frac{\ell}{\ell+1} \varepsilon_{i j k} \partial_{j L-1}\left(\frac{1}{r} S_{k L-1}(u)\right)\right\}, \\
h_{1}^{i j} & =-\frac{4}{c^{4}} \sum_{\ell=2}^{+\infty} \frac{(-)^{\ell}}{\ell !}\left\{\partial_{L-2}\left(\frac{1}{r} \stackrel{(2)}{M}_{i j L-2}(u)\right)+\frac{2 \ell}{\ell+1} \partial_{k L-2}\left(\frac{1}{r} \varepsilon_{k l(i \stackrel{(1)}{S}}{ }_{j(L-2}(u)\right)\right\} .
\end{aligned}
$$

Here, $M_{L}$ and $S_{L}$ denote the so-called "canonical" mass-type and current-type multipole moments of the source, ${ }^{7}$ taken at retarded time $u=t-r$, which agrees in the present approximation with a true null coordinate. Among those moments, the mass monopole $M$ and current dipole $S_{i}$ are constant, while the mass dipole $M_{i}$ varies linearly with time, which means that $\Pi_{i} \equiv M_{i}^{(1)}$ is actually constant. The latter monopole and dipoles thus represent ADM quantities. Now, we need the leading and subleading order terms, proportional to $1 / r$ and $1 / r^{2}$ respectively, in the expansion of $h_{1}^{\mu \nu}$ when $r \rightarrow+\infty$ (with $u=$ const),

$$
h_{1}^{\mu \nu}=\frac{1}{r} z_{1}^{\mu \nu}(\boldsymbol{n}, u)+\frac{1}{r^{2}} y_{1}^{\mu \nu}(\boldsymbol{n}, u)+\mathcal{O}\left(\frac{1}{r^{3}}\right) .
$$

The coefficients $z_{1}^{\mu \nu}$ and $y_{1}^{\mu \nu}$, which depend only on the unit direction $\boldsymbol{n}$ and the retarded time $u$, are explicitly given by

$$
z_{1}^{00}=-4 \sum_{\ell=0}^{+\infty} \frac{n_{L}}{\ell ! c^{\ell+2}} \stackrel{(\ell)}{M_{L}},
$$

\footnotetext{
7 The canonical multipole moments $M_{L}$ and $S_{L}$ differ from the source moments $I_{L}$ and $J_{L}$ by small $2.5 \mathrm{PN}$ corrections (see [23] for details):

$$
M_{L}=I_{L}+\mathcal{O}\left(\frac{1}{c^{5}}\right), \quad S_{L}=J_{L}+\mathcal{O}\left(\frac{1}{c^{5}}\right)
$$
}




$$
\begin{aligned}
& z_{1}^{0 i}=-4 \sum_{\ell=1}^{+\infty} \frac{n_{L-1}}{\ell ! c^{\ell+2}} \stackrel{(\ell)}{M}_{i L-1}+4 \sum_{\ell=1}^{+\infty} \frac{\ell}{(\ell+1) ! c^{\ell+3}} \varepsilon_{i j k} n_{j L-1} \stackrel{(\ell)}{S}_{k L-1}, \\
& z_{1}^{i j}=-4 \sum_{\ell=2}^{+\infty} \frac{n_{L-2}}{\ell ! c^{\ell+2}} \stackrel{(\ell)}{M}_{i j L-2}+8 \sum_{\ell=2}^{+\infty} \frac{\ell}{(\ell+1) ! c^{\ell+3}} n_{k L-2} \varepsilon_{k l(i} \stackrel{(\ell)}{S}_{j) l L-2},
\end{aligned}
$$

and

$$
\begin{aligned}
& y_{1}^{00}=-2 \sum_{\ell=1}^{+\infty} \frac{\ell(\ell+1)}{\ell ! c^{\ell+1}} n_{L} \stackrel{(\ell-1)}{M}_{L} \\
& y_{1}^{0 i}=-2 \sum_{\ell=2}^{+\infty} \frac{(\ell-1) \ell}{\ell ! c^{\ell+1}} n_{L-1} \stackrel{(\ell-1)}{M L-1}_{i L}+2 \sum_{\ell=1}^{+\infty} \frac{\ell^{2}(\ell+1)}{(\ell+1) ! c^{\ell+2}} \varepsilon_{i j k} n_{j L-1} \stackrel{(\ell-1)}{S_{k L-1}}, \\
& y_{1}^{i j}=-2 \sum_{\ell=3}^{+\infty} \frac{(\ell-2)(\ell-1)}{\ell ! c^{\ell+1}} n_{L-2} \stackrel{(\ell-1)}{M_{i j L-2}}+4 \sum_{\ell=2}^{+\infty} \frac{(\ell-1) \ell^{2}}{(\ell+1) ! c^{\ell+2}} n_{k L-2} \varepsilon_{k l(i}^{(\ell-1)} S_{j) l L-2}^{(\ell)} .
\end{aligned}
$$

We plug these expressions into the quadratic source $\Lambda_{2}^{\mu \nu}$ to control the leading and subleading terms, behaving as $1 / r^{2}$ and $1 / r^{3}$ respectively, of its asymptotic expansion in powers of $1 / r$ at null infinity

$$
\Lambda_{2}^{\mu \nu}=\frac{1}{r^{2}} Q_{2}^{\mu \nu}(\boldsymbol{n}, u)+\frac{1}{r^{3}} R_{2}^{\mu \nu}(\boldsymbol{n}, u)+\mathcal{O}\left(\frac{1}{r^{4}}\right) .
$$

Inserting then these expansions into the fluxes (4.4) and integrating over a sphere at infinity (hence $\mathrm{d} S_{i}=\mathrm{d} \Omega n_{i} r^{2}$ with $\mathrm{d} \Omega$ representing the element of solid angle) yields

$$
\begin{aligned}
\frac{\mathrm{d} E}{\mathrm{~d} u} & =-\frac{G c^{5}}{16 \pi} \oint_{\mathscr{S}} \mathrm{d} \Omega n_{i} Q_{2}^{0 i}+\mathcal{O}\left(\frac{1}{r}\right)+\mathcal{O}\left(G^{2}\right) \\
\frac{\mathrm{d} P^{i}}{\mathrm{~d} u} & =-\frac{G c^{4}}{16 \pi} \oint_{\mathscr{S}} \mathrm{d} \Omega n_{j} Q_{2}^{i j}+\mathcal{O}\left(\frac{1}{r}\right)+\mathcal{O}\left(G^{2}\right) \\
\frac{\mathrm{d} J_{i}}{\mathrm{~d} u} & =-\frac{G c^{4}}{16 \pi} \varepsilon_{i j k} \oint_{\mathscr{S}} \mathrm{d} \Omega n_{j} n_{l}\left[r Q_{2}^{k l}+R_{2}^{k l}\right]+\mathcal{O}\left(\frac{1}{r}\right)+\mathcal{O}\left(G^{2}\right) \\
\frac{\mathrm{d} G_{i}}{\mathrm{~d} u} & =P_{i}-\frac{G c^{3}}{16 \pi} \oint_{\mathscr{S}} \mathrm{d} \Omega n_{j}\left[r\left(n_{i} Q_{2}^{0 j}-Q_{2}^{i j}\right)+\left(n_{i} R_{2}^{0 j}-R_{2}^{i j}\right)\right]+\mathcal{O}\left(\frac{1}{r}\right)+\mathcal{O}\left(G^{2}\right)
\end{aligned}
$$

Importantly, for the fluxes of angular momentum $J_{i}$ and center-of-mass position $G_{i}$, the leading term formally behaves like $r$, implying that the fluxes a priori diverge at infinity. However, we shall find that those divergent contributions vanish after angular integration, modulo total time derivatives which reduce to zero in the center-of-mass frame and can be removed by means of an appropriate redefinition of $J_{i}$ and $G_{i}$ (see the footnote 9). This fact is well known in the case of the angular momentum and we will verify it explicitly in the case of the center-of-mass position.

To proceed further, we denote by $k^{\mu}=(1, \boldsymbol{n})$ a Minkowskian null vector [with thus $\left.k_{\nu}=(-1, \boldsymbol{n})\right]$, by $n^{\mu}=(0, \boldsymbol{n})$ the corresponding purely spatial vector, and by $\delta_{\nu}=\left(0, \delta_{i}\right)$ an operator purely acting on angles and whose space part is defined as $\delta_{i}=r \partial_{i} n_{j} \frac{\partial}{\partial n^{j}}=\perp_{i j} \frac{\partial}{\partial n^{j}}$, where $\perp_{i j}=\delta_{i j}-n_{i} n_{j}$ is the orthogonal projector onto the plane perpendicular to $n_{i}$. The 
following relations then hold: $k^{\mu} k_{\mu}=0, n^{\mu} k_{\mu}=1$ and $k^{\mu} \delta_{\mu}=n^{\mu} \delta_{\mu}=0$. Moreover, the tensor $\delta_{\mu} k_{\nu}$ is actually symmetric, i.e., $\delta_{\mu} k_{\nu}=\delta_{\nu} k_{\mu}$, since its $i j$ components are given by $\perp_{i j}$ whereas its other components are zero. With those notations, the harmonic-gauge condition at the linearized order, $\partial_{\nu} h_{1}^{\mu \nu}=0$, implies the constraints

$$
\begin{aligned}
& k_{\nu} \stackrel{(1)}{z}_{1}^{\mu \nu}=0, \\
& k_{\nu}{ }^{(1)}{ }_{1}^{\mu \nu}=\left(\delta_{\nu}-n_{\nu}\right) z_{1}^{\mu \nu} .
\end{aligned}
$$

After inserting the expressions (4.9)-(4.10) into the quadratic source $\Lambda_{2}$ of the Einstein field equations, we readily obtain the leading order coefficient of $1 / r^{2}$ when $r \rightarrow+\infty$ [see Eq. (4.11)] as

$$
Q_{2}^{\mu \nu}=-4 \frac{k^{\rho} \prod_{\rho}}{c^{5}} \stackrel{(2)}{z_{1}} \mu \nu+\frac{k^{\mu} k^{\nu}}{c^{2}}\left(\frac{1}{2} \stackrel{(1)}{z}_{1} \rho \sigma \stackrel{(1)}{z_{1 \rho \sigma}}-\frac{1}{4} \stackrel{(1)}{z_{1 \rho} \rho} \stackrel{(1)}{z_{1 \sigma}}\right)
$$

where $\Pi_{\rho}=\left(M c, \Pi_{i}\right)$ with $\Pi_{i}=M_{i}^{(1)}$ denotes the constant (ADM) linear four-momentum. The quantity in parenthesis in the second term is proportional to the gravitational-wave energy flux (at quadratic order). The next order piece, $\propto 1 / r^{3}$, in the quadratic source $\Lambda_{2}$ is more involved:

$$
\begin{aligned}
& R_{2}^{\mu \nu}=-4 \frac{k^{\rho} \Pi_{\rho}}{c^{5}} \stackrel{(2)}{y}_{1}^{\mu \nu}-8 \frac{n^{\rho} \prod_{\rho}}{c^{5}} \stackrel{(1)}{z}_{1}^{\mu \nu}+8 \Pi^{\rho} \delta_{\rho} \stackrel{(1)}{z}_{1}^{\mu \nu}+4 \eta^{\mu \nu} n_{\rho} \Pi_{\sigma} \stackrel{(1)}{z}_{1}^{\rho \sigma} \\
& +8 \stackrel{(1)}{z}_{1}^{\rho(\mu}\left(\Pi^{\nu)} n_{\rho}-n^{\nu)} \Pi_{\rho}\right)+\delta_{\rho} k_{\sigma} z^{\rho \sigma}{\stackrel{(1)}{z_{1}}}^{\mu \nu}-k_{\rho} k_{\sigma} y^{\rho \sigma}{\stackrel{(2)}{z_{1}}}^{\mu \nu}+\eta^{\mu \nu} \delta_{\rho} k_{\sigma}{\stackrel{(1)}{z_{1}}}^{\rho \lambda} z_{1 \lambda}^{\sigma} \\
& -\eta^{\mu \nu}\left(\frac{1}{2} \stackrel{(1)}{z}_{1}^{\rho \sigma} z_{1 \rho \sigma}-\frac{1}{4} \stackrel{(1)}{z}_{1 \rho}^{\rho} z_{1 \sigma}^{\sigma}\right)+k^{\mu} k^{\nu}\left(\stackrel{(1)}{z}_{1}^{\rho \sigma} \stackrel{(1)}{y}_{1 \rho \sigma}-\frac{1}{2} \stackrel{(1)}{z}_{1 \rho}^{\rho} \stackrel{(1)}{y_{1 \sigma}^{\sigma}}\right)+\frac{1}{2} \stackrel{(1)}{z}_{1 \rho}^{\rho} k^{(\mu} \delta^{\nu)} z_{1 \sigma}^{\sigma} \\
& \left.+k^{(\mu} n^{\nu)}\left(\stackrel{(1)}{z}_{1}^{\rho \sigma} z_{1 \rho \sigma}-\frac{1}{2} \stackrel{(1)}{z}_{1 \rho}^{\rho} z_{1 \sigma}^{\sigma}\right)+2 \delta_{\rho} k_{\sigma} \stackrel{(1)}{z}{ }_{1}^{\rho(\mu} z_{1}^{\nu) \sigma}+2 \stackrel{(1)}{z_{1}} \rho(\mu) z_{1 \rho}^{\nu)}-2 \delta^{(\mu} k_{\rho} \stackrel{(1)}{z_{1}} \nu\right){ }_{1 \sigma}^{\rho} \\
& -\stackrel{(1)}{z}_{1}^{\rho \sigma} k^{(\mu} \delta^{\nu)} z_{1 \rho \sigma}+2 k^{(\mu} \stackrel{(1)}{z}_{1}^{\rho \sigma} \delta_{\rho} z_{1 \sigma}^{\nu)}-2 k^{(\mu} \stackrel{(1)}{z}_{1 \rho \sigma} n^{\rho} z_{1}^{\nu) \sigma}-2 \stackrel{(1)}{z}_{1}^{\rho(\mu} k^{\nu)}\left(\delta_{\sigma}-n_{\sigma}\right) z_{1 \rho}^{\sigma} .
\end{aligned}
$$

As a check of those expressions, due to the fact that $\partial_{\nu} \Lambda_{2}^{\mu \nu}=0$, we must have

$$
\begin{aligned}
& k_{\nu} \stackrel{(1)}{Q}_{2}^{\mu \nu}=0, \\
& k_{\nu} \stackrel{(1)}{R}_{2}^{\mu \nu}=\left(\delta_{\nu}-2 n_{\nu}\right) Q_{2}^{\mu \nu} .
\end{aligned}
$$

The final steps consist of replacing $Q_{2}^{\mu \nu}$ and $R_{2}^{\mu \nu}$ by their expressions (4.14)-(4.15) in Eqs. (4.12), to perform the angular integration and let the surface $\mathscr{S}$ tend towards $\mathscr{I}^{+}$. For the fluxes of $E$ and $P_{i}$, the computation is straightforward and not too long, leading to the well-known multipolar series parametrized by the (canonical) mass and current multipole moments $M_{L}$ and $S_{L}$ :

$$
\begin{aligned}
\frac{\mathrm{d} E}{\mathrm{~d} u}=-\sum_{\ell=2}^{+\infty} \frac{G}{c^{2 \ell+1}} & \left\{\frac{(\ell+1)(\ell+2)}{(\ell-1) \ell \ell !(2 \ell+1) ! !} \stackrel{(\ell+1)(\ell+1)}{M_{L}} M_{L}\right. \\
& \left.+\frac{4 \ell(\ell+2)}{c^{2}(\ell-1)(\ell+1) !(2 \ell+1) ! !} \stackrel{(\ell+1)(\ell+1)}{S}_{L} S_{L}\right\}+\mathcal{O}\left(G^{2}\right),
\end{aligned}
$$




$$
\begin{aligned}
\frac{\mathrm{d} P_{i}}{\mathrm{~d} u}=-\sum_{\ell=2}^{+\infty} \frac{G}{c^{2 \ell+3}} & \left\{\frac{2(\ell+2)(\ell+3)}{\ell(\ell+1) !(2 \ell+3) ! !} \stackrel{(\ell+2)}{M_{i L}} \stackrel{(\ell+1)}{M_{L}}+\frac{8(\ell+2)}{(\ell-1)(\ell+1) !(2 \ell+1) ! !} \varepsilon_{i j k} \stackrel{(\ell+1)}{M_{j L-1}} \stackrel{(\ell+1)}{S_{k L-1}}\right. \\
& \left.+\frac{8(\ell+3)}{c^{2}(\ell+1) !(2 \ell+3) ! !} \stackrel{(\ell+2)(\ell+1)}{S}_{i L}^{S_{L}}\right\}+\mathcal{O}\left(G^{2}\right) .
\end{aligned}
$$

Here the moments are evaluated at the retarded time $u$, which becomes a retarded null coordinate with the present approximation, where contributions to $\tau_{\mathrm{GW}}^{\mu \nu}$ beyond quadratic order in $G$ are ignored. In the case of the linear momentum we discard total time derivatives since they can be transferred to the LHS of the balance equations.

Concerning the fluxes of $J_{i}$ and $G_{i}$, the calculations are more involved because of the more complicated structure of the term $R_{2}$ given by (4.15). ${ }^{8}$ Furthermore, we have to check that the formally divergent parts of the fluxes when $r \rightarrow+\infty$ [terms involving an explicit factor $r$ in $(4.12 \mathrm{c})$ and $(4.12 \mathrm{~d})$ ] actually reduce to total time derivatives after angular averaging. In fact, we find the very satisfying result that all the divergent terms cancel out after the angular integration when the source is at rest, $\Pi_{i}=0 .{ }^{9}$ Once this verification has been done, supplemented with an appropriate redefinition of $J_{i}$ and $G_{i}$ in the LHS, we can take the limit $r \rightarrow+\infty$. We find

$$
\begin{aligned}
\frac{\mathrm{d} J_{i}}{\mathrm{~d} u}=-\varepsilon_{i j k} \sum_{\ell=2}^{+\infty} \frac{G}{c^{2 \ell+1}}\left\{\frac{(\ell+1)(\ell+2)}{(\ell-1) \ell !(2 \ell+1) ! !} \stackrel{(\ell)}{M}_{j L-1} \stackrel{(\ell+1)}{M_{k L-1}}\right. \\
\left.+\frac{4 \ell^{2}(\ell+2)}{c^{2}(\ell-1)(\ell+1) !(2 \ell+1) ! !} \stackrel{(\ell)}{S}_{j L-1}^{\stackrel{(\ell+1)}{S}}{ }_{k L-1}\right\}+\mathcal{O}\left(G^{2}\right), \\
\frac{\mathrm{d} G_{i}}{\mathrm{~d} u}=P_{i}-\sum_{\ell=2}^{+\infty} \frac{G}{c^{2 \ell+3}}\left\{\frac{2(\ell+2)(\ell+3)}{\ell \ell !(2 \ell+3) ! !} \stackrel{(\ell+1)}{M_{i L}} \stackrel{(\ell+1)}{M_{L}}+\frac{8(\ell+3)}{c^{2} \ell !(2 \ell+3) ! !} \stackrel{(\ell+1)}{S_{i L}} \stackrel{(\ell+1)}{S_{L}}\right\}+\mathcal{O}\left(G^{2}\right) .
\end{aligned}
$$

As we stressed several times, the formulas (4.17)-(4.18) are approximate, but the multipole moments $M_{L}$ and $S_{L}$ therein can be related to the matter source in a precise way; in particular they differ from the source moments $I_{L}$ and $J_{L}$ by small 2.5PN corrections. The next-order level $\mathcal{O}\left(G^{2}\right)$ contains the tail effect, which can be included if necessary into the definition of the radiative multipole moments $U_{L}$ and $V_{L}$ measured at $\mathscr{I}^{+}$. An alternative derivation of the fluxes, directly in terms of the radiative moments $U_{L}$ and $V_{L}$, should

8 The explicit computations of the fluxes entering the RHS of (4.12) leading to Eqs. (4.17)-(4.18) have been performed with the software Mathematica ${ }^{\circledR}$.

${ }^{9}$ When the source is moving with respect to the asymptotic rest frame (i.e., $\Pi_{i} \neq 0$ ), we find some remaining divergent terms, but only in the form of a total time derivative. These terms correct the angular momentum $J_{i}$ and center of mass $G_{i}$ in the LHS by the quantities:

$$
\begin{aligned}
\delta J_{i}^{\mathrm{div}} & =\frac{4 G}{15 c^{6}} r \stackrel{(3)}{S}_{i j} \Pi_{j}, \\
\delta G_{i}^{\mathrm{div}} & =-\frac{2 G}{5 c^{6}} r \stackrel{(3)}{M}_{i j} \Pi_{j} .
\end{aligned}
$$

We leave to future work the task of investigating how these divergent terms should combine with other divergences in the definitions of $J_{i}$ and $G_{i}$ [Eqs. (4.5c) and (4.5d)] to yield a finite result. 
yield the same structure as in (4.17)-(4.18), but with the replacements $M_{L}^{(\ell)} \rightarrow U_{L}$ and $S_{L}^{(\ell)} \rightarrow V_{L}$. The formula (4.18a) for the angular momentum (or, rather, its equivalent in terms of radiative moments) is already known (see notably [29]). ${ }^{10}$

As for the formula (4.18b) regarding the center of mass position, it does not seem to have appeared previously in the literature. For $\ell=2$, we recover the dominant mass-type contribution (1.4), with the correct coefficient $2 / 21$ derived by means of a radiation-reaction calculation in Sec. (III). This coefficient has also been found in Ref. [59], as well as the dominant current-type contribution in the case $\ell=2$, in agreement with our formula (4.18b) (modulo a time derivative). We shall now present still another confirmation of the leading effect (1.4) by a radiation-reaction calculation but, this time, restricted to the case of compact binary systems.

\section{RADIATION-REACTION FORCE FOR COMPACT BINARY SYSTEMS}

The radiation-reaction force on compact binary systems at the $3.5 \mathrm{PN}$ order has been investigated in many works. It was computed in a large class of coordinate systems, though limiting oneself to the frame of the center of mass, as a consequence of the energy and angular momentum balance equations [26, 27]. On the other hand, using various approaches and specific coordinate systems, it was established in a general frame from first principles, i.e., not relying on any balance equation [64-68]. All these works consistently agree, as they do with the general fluid formalism described in Sec. II.

The two mass components are referred to as $m_{1}$ and $m_{2}$ henceforth. We denote by $r_{12}=\left|\boldsymbol{y}_{1}-\boldsymbol{y}_{2}\right|$ the harmonic-coordinate distance between the two particles $\left\{\boldsymbol{y}_{1}, \boldsymbol{y}_{2}\right\}$, by $\boldsymbol{n}_{12}=\left(\boldsymbol{y}_{1}-\boldsymbol{y}_{2}\right) / r_{12}$ the corresponding unit direction, by $\boldsymbol{v}_{1}=\mathrm{d} \boldsymbol{y}_{1} / \mathrm{d} t$ and $\boldsymbol{a}_{1}=\mathrm{d} \boldsymbol{v}_{1} / \mathrm{d} t$ the coordinate velocity and acceleration of the particle 1 , respectively (idem for particle 2 ). We shall also occasionally use the notation $\boldsymbol{v}_{12}=\boldsymbol{v}_{1}-\boldsymbol{v}_{2}$ for the relative velocity. The Euclidean scalar product of vectors is denoted with parentheses, e.g. $\left(n_{12} v_{1}\right)=\boldsymbol{n}_{12} \cdot \boldsymbol{v}_{1}$. The $3.5 \mathrm{PN}$ acceleration of the particle 1 can then be written, for general orbits in a general harmonic-coordinate system, as

$$
\boldsymbol{a}_{1}=\boldsymbol{a}_{1}^{\mathrm{N}}+\frac{1}{c^{2}} \boldsymbol{a}_{1}^{1 \mathrm{PN}}+\frac{1}{c^{4}} \boldsymbol{a}_{1}^{2 \mathrm{PN}}+\frac{1}{c^{5}} \boldsymbol{a}_{1}^{2.5 \mathrm{PN}}+\frac{1}{c^{6}} \boldsymbol{a}_{1}^{3 \mathrm{PN}}+\frac{1}{c^{7}} \boldsymbol{a}_{1}^{3.5 \mathrm{PN}}+\mathcal{O}\left(\frac{1}{c^{8}}\right)
$$

The conservative part of the acceleration is actually known up to the 4PN order [62]. The dissipative radiation-reaction part of interest here reads, at 2.5PN and 3.5PN orders,

$$
\begin{aligned}
\boldsymbol{a}_{1}^{2.5 \mathrm{PN}}=\frac{4 G^{2} m_{1} m_{2}}{5 r_{12}^{3}} & \left(\left(n_{12} v_{12}\right)\left[-6 \frac{G m_{1}}{r_{12}}+\frac{52}{3} \frac{G m_{2}}{r_{12}}+3 v_{12}^{2}\right] \boldsymbol{n}_{12}\right. \\
+ & {\left.\left[2 \frac{G m_{1}}{r_{12}}-8 \frac{G m_{2}}{r_{12}}-v_{12}^{2}\right] \boldsymbol{v}_{12}\right) }
\end{aligned}
$$

10 This formula has been provided by Thorne [29] (who refers also to an unpublished calculation by DeWitt) without details. Here, we have tried to be more comprehensive by providing in Eq. (4.15) the explicit expression of the crucial $1 / r^{3}$ piece of the GW pseudo tensor. See Ref. [63] for a recent investigation of the angular momentum flux. 


$$
\begin{aligned}
& \boldsymbol{a}_{1}^{3.5 \mathrm{PN}}=\frac{G^{2} m_{1} m_{2}}{r_{12}^{3}}\left\{\frac{G^{2} m_{1}^{2}}{r_{12}^{2}}\left[\left(\frac{3992}{105}\left(n_{12} v_{1}\right)-\frac{4328}{105}\left(n_{12} v_{2}\right)\right) \boldsymbol{n}_{12}-\frac{184}{21} \boldsymbol{v}_{12}\right]\right. \\
& +\frac{G^{2} m_{1} m_{2}}{r_{12}^{3}}\left[\left(-\frac{13576}{105}\left(n_{12} v_{1}\right)+\frac{2872}{21}\left(n_{12} v_{2}\right)\right) \boldsymbol{n}_{12}+\frac{6224}{105} \boldsymbol{v}_{12}\right] \\
& +\frac{G^{2} m_{2}^{2}}{r_{12}^{3}}\left[-\frac{3172}{21}\left(n_{12} v_{12}\right) \boldsymbol{n}_{12}+\frac{6388}{105} \boldsymbol{v}_{12}\right] \\
& +\frac{G m_{1}}{r_{12}}\left[\left(48\left(n_{12} v_{1}\right)^{3}-\frac{696}{5}\left(n_{12} v_{1}\right)^{2}\left(n_{12} v_{2}\right)+\frac{744}{5}\left(n_{12} v_{1}\right)\left(n_{12} v_{2}\right)^{2}\right.\right. \\
& -\frac{288}{5}\left(n_{12} v_{2}\right)^{3}-\frac{4888}{105}\left(n_{12} v_{1}\right) v_{1}^{2}+\frac{5056}{105}\left(n_{12} v_{2}\right) v_{1}^{2} \\
& +\frac{2056}{21}\left(n_{12} v_{1}\right)\left(v_{1} v_{2}\right)-\frac{2224}{21}\left(n_{12} v_{2}\right)\left(v_{1} v_{2}\right) \\
& \left.-\frac{1028}{21}\left(n_{12} v_{1}\right) v_{2}^{2}+\frac{5812}{105}\left(n_{12} v_{2}\right) v_{2}^{2}\right) \boldsymbol{n}_{12} \\
& +\left(\frac{52}{15}\left(n_{12} v_{1}\right)^{2}-\frac{56}{15}\left(n_{12} v_{1}\right)\left(n_{12} v_{2}\right)-\frac{44}{15}\left(n_{12} v_{2}\right)^{2}-\frac{132}{35} v_{1}^{2}\right. \\
& \left.\left.+\frac{152}{35}\left(v_{1} v_{2}\right)-\frac{48}{35} v_{2}^{2}\right) \boldsymbol{v}_{12}\right] \\
& +\frac{G m_{2}}{r_{12}}\left[\left(-\frac{582}{5}\left(n_{12} v_{1}\right)^{3}+\frac{1746}{5}\left(n_{12} v_{1}\right)^{2}\left(n_{12} v_{2}\right)-\frac{1954}{5}\left(n_{12} v_{1}\right)\left(n_{12} v_{2}\right)^{2}\right.\right. \\
& +158\left(n_{12} v_{2}\right)^{3}+\frac{3568}{105}\left(n_{12} v_{12}\right)\left(v_{1} v_{1}\right)-\frac{2864}{35}\left(n_{12} v_{1}\right)\left(v_{1} v_{2}\right) \\
& \left.+\frac{10048}{105}\left(n_{12} v_{2}\right)\left(v_{1} v_{2}\right)+\frac{1432}{35}\left(n_{12} v_{1}\right) v_{2}^{2}-\frac{5752}{105}\left(n_{12} v_{2}\right) v_{2}^{2}\right) \boldsymbol{n}_{12} \\
& +\left(\frac{454}{15}\left(n_{12} v_{1}\right)^{2}-\frac{372}{5}\left(n_{12} v_{1}\right)\left(n_{12} v_{2}\right)+\frac{854}{15}\left(n_{12} v_{2}\right)^{2}-\frac{152}{21} v_{1}^{2}\right. \\
& \left.\left.+\frac{2864}{105}\left(v_{1} v_{2}\right)-\frac{1768}{105} v_{2}^{2}\right) \boldsymbol{v}_{12}\right] \\
& +\left(-56\left(n_{12} v_{12}\right)^{5}+60\left(n_{12} v_{1}\right)^{3} v_{12}^{2}-180\left(n_{12} v_{1}\right)^{2}\left(n_{12} v_{2}\right) v_{12}^{2}+174\left(n_{12} v_{1}\right)\left(n_{12} v_{2}\right)^{2} v_{12}^{2}\right. \\
& -54\left(n_{12} v_{2}\right)^{3} v_{12}^{2}-\frac{246}{35}\left(n_{12} v_{12}\right) v_{1}^{4}+\frac{1068}{35}\left(n_{12} v_{1}\right) v_{1}^{2}\left(v_{1} v_{2}\right) \\
& -\frac{984}{35}\left(n_{12} v_{2}\right) v_{1}^{2}\left(v_{1} v_{2}\right)-\frac{1068}{35}\left(n_{12} v_{1}\right)\left(v_{1} v_{2}\right)^{2}+\frac{180}{7}\left(n_{12} v_{2}\right)\left(v_{1} v_{2}\right)^{2} \\
& -\frac{534}{35}\left(n_{12} v_{1}\right) v_{1}^{2} v_{2}^{2}+\frac{90}{7}\left(n_{12} v_{2}\right) v_{1}^{2} v_{2}^{2}+\frac{984}{35}\left(n_{12} v_{1}\right)\left(v_{1} v_{2}\right) v_{2}^{2} \\
& \left.-\frac{732}{35}\left(n_{12} v_{2}\right)\left(v_{1} v_{2}\right) v_{2}^{2}-\frac{204}{35}\left(n_{12} v_{1}\right) v_{2}^{4}+\frac{24}{7}\left(n_{12} v_{2}\right) v_{2}^{4}\right) \boldsymbol{n}_{12} \\
& +\left(60\left(n_{12} v_{12}\right)^{4}-\frac{348}{5}\left(n_{12} v_{1}\right)^{2} v_{12}^{2}+\frac{684}{5}\left(n_{12} v_{1}\right)\left(n_{12} v_{2}\right) v_{12}^{2}-66\left(n_{12} v_{2}\right)^{2} v_{12}^{2}\right. \\
& +\frac{334}{35} v_{1}^{4}-\frac{1336}{35} v_{1}^{2}\left(v_{1} v_{2}\right)+\frac{1308}{35}\left(v_{1} v_{2}\right)^{2}+\frac{654}{35} v_{1}^{2} v_{2}^{2}
\end{aligned}
$$




$$
\left.\left.-\frac{1252}{35}\left(v_{1} v_{2}\right) v_{2}^{2}+\frac{292}{35} v_{2}^{4}\right) \boldsymbol{v}_{12}\right\} .
$$

With this acceleration in hand, it is straightforward to derive the balance equations. Consistently with the accuracy of (5.2), we can work out the 1PN relative equations for energy and angular momentum [see (3.20)] as well as the Newtonian relative ones for linear momentum and center-of-mass position [see (3.9) and (3.17)]. The test we have made consists in verifying that, for each of these quantities, there exist some radiation-reaction terms in the form of total time derivatives that will contribute to the LHS of the balance equations, with the expected fluxes in the RHS. In fact, the unique existence of these terms (in the particular coordinate system we are working with) is necessary and sufficient to prove the correctness of the balance equations for the binary systems.

Let us apply the method to the linear momentum $P_{i}$ and center of mass position $G_{i}$, in order to check the coefficient 2/21 in front of the new flux term in Eq. (3.17). We want thus to construct some total time derivatives so that, as a consequence of the radiation-reaction force (5.2), the balance equations (3.9) and (3.17) are satisfied. We indeed find well defined expressions for the linear momentum and center-of-mass position,

$$
\begin{aligned}
& \mathbf{P}=\mathbf{P}^{\mathrm{N}}+\frac{1}{c^{2}} \mathbf{P}^{1 \mathrm{PN}}+\frac{1}{c^{4}} \mathbf{P}^{2 \mathrm{PN}}+\frac{1}{c^{5}} \mathbf{P}^{2.5 \mathrm{PN}}+\frac{1}{c^{6}} \mathbf{P}^{3 \mathrm{PN}}+\frac{1}{c^{7}} \mathbf{P}^{3.5 \mathrm{PN}}+\mathcal{O}\left(\frac{1}{c^{8}}\right), \\
& \mathbf{G}=\mathbf{G}^{\mathrm{N}}+\frac{1}{c^{2}} \mathbf{G}^{1 \mathrm{PN}}+\frac{1}{c^{4}} \mathbf{G}^{2 \mathrm{PN}}+\frac{1}{c^{5}} \mathbf{G}^{2.5 \mathrm{PN}}+\frac{1}{c^{6}} \mathbf{G}^{3 \mathrm{PN}}+\frac{1}{c^{7}} \mathbf{G}^{3.5 \mathrm{PN}}+\mathcal{O}\left(\frac{1}{c^{8}}\right),
\end{aligned}
$$

in which the $2.5 \mathrm{PN}$ and $3.5 \mathrm{PN}$ radiation-reaction terms are uniquely determined as

$$
\begin{aligned}
\mathbf{P}^{2.5 \mathrm{PN}}= & \frac{4 G^{2} m_{1}^{2} m_{2}}{5 r_{12}^{2}}\left(v_{12}^{2}-\frac{2 G m_{1}}{r_{12}}\right) \boldsymbol{n}_{12}+1 \leftrightarrow 2 \\
\mathbf{P}^{3.5 \mathrm{PN}}= & \left\{\frac{288}{35} \frac{G^{4} m_{1}^{4} m_{2}}{r_{12}^{4}}+\frac{332}{35} \frac{G^{4} m_{1}^{3} m_{2}^{2}}{r_{12}^{4}}+\frac{92}{15} \frac{G^{3} m_{1}^{2} m_{2}^{2}}{r_{12}^{3}}\left(n_{12} v_{1}\right)^{2}\right. \\
& +\frac{G^{3} m_{1}^{3} m_{2}}{r_{12}^{3}}\left(\frac{152}{15}\left(n_{12} v_{1}\right)^{2}-\frac{56}{3}\left(n_{12} v_{1}\right)\left(n_{12} v_{2}\right)+\frac{576}{35}\left(v_{1} v_{2}\right)-\frac{288}{35} v_{1}^{2}\right) \\
& +\frac{G^{3} m_{1} m_{2}^{3}}{r_{12}^{3}}\left(-\frac{176}{15}\left(n_{12} v_{1}\right)^{2}+\frac{288}{35} v_{1}^{2}\right) \\
& +\frac{G^{3} m_{1}^{2} m_{2}^{2}}{r_{12}^{4}}\left(-\frac{8}{35}\left(n_{12} v_{1}\right)^{2}\left(n_{12} y_{1}\right)+\frac{16}{35}\left(n_{12} v_{1}\right)\left(n_{12} v_{2}\right)\left(n_{12} y_{1}\right)-\frac{8}{35}\left(n_{12} v_{2}\right)^{2}\left(n_{12} y_{1}\right)\right. \\
& -\frac{64}{35}\left(v_{1} v_{2}\right)\left(n_{12} y_{1}\right)-\frac{16}{21}\left(n_{12} v_{1}\right)\left(v_{1} y_{1}\right)+\frac{16}{21}\left(n_{12} v_{2}\right)\left(v_{1} y_{1}\right)+\frac{16}{21}\left(n_{12} v_{1}\right)\left(v_{2} y_{1}\right) \\
& \left.+\frac{16}{21}\left(n_{12} v_{2}\right)\left(v_{2} y_{1}\right)+\frac{32}{35}\left(n_{12} y_{1}\right) v_{1}^{2}+\frac{32}{35}\left(n_{12} y_{2}\right) v_{1}^{2}\right) \\
& \quad+\frac{G_{1}^{2} m_{2}^{2}}{r_{12}^{2}}\left(8\left(n_{12} v_{1}\right)^{4}-32\left(n_{12} v_{1}\right)^{3}\left(n_{12} v_{2}\right)+\frac{44}{5}\left(n_{12} v_{1}\right)^{2}\left(v_{1} v_{2}\right)-\frac{22}{5}\left(n_{12} v_{1}\right)^{2} v_{1}^{2}\right. \\
& +\frac{G^{2} m_{1}^{2} m_{2}}{r_{12}^{2}}\left(-8\left(n_{12} v_{1}\right)^{4}+32\left(n_{12} v_{2}\right) v_{1}^{2}-\frac{28}{5}\left(n_{12} v_{2}\right)^{2} v_{1}^{2}+\frac{584}{105}\left(v_{1} v_{2}\right) v_{1}^{2}-\frac{188}{105} v_{1}^{4}\right) \\
& +\frac{112}{5}\left(n_{12} v_{2}\right)-48\left(n_{12} v_{1}\right)^{2}\left(n_{12} v_{2}\right)^{2}-\frac{56}{5}\left(n_{12} v_{1}\right)^{2}\left(v_{1} v_{2}\right) \\
& \left.+v_{1} v_{2}\right)+\frac{100}{21}\left(v_{1} v_{2}\right)^{2}+\frac{28}{5}\left(n_{12} v_{1}\right)^{2} v_{1}^{2}-\frac{56}{5}\left(n_{12} v_{1}\right)\left(n_{12} v_{2}\right) v_{1}^{2}
\end{aligned}
$$




$$
\begin{aligned}
& \left.\left.+\frac{22}{5}\left(n_{12} v_{2}\right)^{2} v_{1}^{2}-\frac{584}{105}\left(v_{1} v_{2}\right) v_{1}^{2}+\frac{188}{105} v_{1}^{4}+\frac{292}{105} v_{1}^{2} v_{2}^{2}\right)\right\} \boldsymbol{n}_{12} \\
+ & \left\{\frac{G^{3} m_{1}^{2} m_{2}^{2}}{r_{12}^{3}}\left(-\frac{12}{5}\left(n_{12} v_{1}\right)-4\left(n_{12} v_{2}\right)\right)+\frac{G^{3} m_{1}^{3} m_{2}}{r_{12}^{3}}\left(-\frac{8}{15}\left(n_{12} v_{1}\right)+\frac{8}{15}\left(n_{12} v_{2}\right)\right)\right. \\
+ & \frac{G^{3} m_{1} m_{2}^{3}}{r_{12}^{3}}\left(\frac{8}{15}\left(n_{12} v_{1}\right)+\frac{16}{15}\left(n_{12} v_{2}\right)\right) \\
+ & \frac{G^{3} m_{1}^{2} m_{2}^{2}}{r_{12}^{4}}\left(-\frac{16}{21}\left(n_{12} v_{1}\right)\left(n_{12} y_{1}\right)+\frac{16}{21}\left(n_{12} v_{2}\right)\left(n_{12} y_{1}\right)+\frac{32}{35}\left(v_{1} y_{1}\right)-\frac{32}{35}\left(v_{2} y_{1}\right)\right. \\
& \left.-\frac{16}{21}\left(n_{12} v_{1}\right)\left(n_{12} y_{2}\right)+\frac{16}{21}\left(n_{12} v_{2}\right)\left(n_{12} y_{2}\right)+\frac{32}{35}\left(v_{1} y_{2}\right)-\frac{32}{35}\left(v_{2} y_{2}\right)\right) \\
+ & \frac{G^{2} m_{1} m_{2}^{2}}{r_{12}^{2}}\left(-\frac{52}{5}\left(n_{12} v_{1}\right)^{3}+\frac{156}{5}\left(n_{12} v_{1}\right)^{2}\left(n_{12} v_{2}\right)-\frac{156}{5}\left(n_{12} v_{1}\right)\left(n_{12} v_{2}\right)^{2}+\frac{52}{5}\left(n_{12} v_{2}\right)^{3}\right. \\
& -\frac{304}{15}\left(n_{12} v_{1}\right)\left(v_{1} v_{2}\right)+\frac{328}{15}\left(n_{12} v_{2}\right)\left(v_{1} v_{2}\right)+\frac{152}{15}\left(n_{12} v_{1}\right) v_{1}^{2}-\frac{164}{15}\left(n_{12} v_{2}\right) v_{1}^{2} \\
& \left.+\frac{152}{15}\left(n_{12} v_{1}\right) v_{2}^{2}-\frac{164}{15}\left(n_{12} v_{2}\right) v_{2}^{2}\right) \\
+ & \frac{G^{2} m_{1}^{2} m_{2}}{r_{12}^{2}}\left(\frac{52}{5}\left(n_{12} v_{1}\right)^{3}-\frac{156}{5}\left(n_{12} v_{1}\right)^{2}\left(n_{12} v_{2}\right)+\frac{156}{5}\left(n_{12} v_{1}\right)\left(n_{12} v_{2}\right)^{2}-\frac{52}{5}\left(n_{12} v_{2}\right)^{3}\right. \\
& +\frac{304}{15}\left(n_{12} v_{1}\right)\left(v_{1} v_{2}\right)-\frac{304}{15}\left(n_{12} v_{2}\right)\left(v_{1} v_{2}\right)-\frac{152}{15}\left(n_{12} v_{1}\right) v_{1}^{2}+\frac{152}{15}\left(n_{12} v_{2}\right) v_{1}^{2} \\
& \left.\left.-\frac{152}{15}\left(n_{12} v_{1}\right) v_{2}^{2}+\frac{152}{15}\left(n_{12} v_{2}\right) v_{2}^{2}\right)\right\} \boldsymbol{v}_{1} \\
+ & \frac{G^{3} m_{1}^{2} m_{2}^{2}}{r_{12}^{4}}\left(\frac{164}{105}\left(n_{12} v_{1}\right)^{2}-\frac{328}{105}\left(n_{12} v_{1}\right)\left(n_{12} v_{2}\right)+\frac{164}{105}\left(n_{12} v_{2}\right)^{2}+\frac{352}{105}\left(v_{1} v_{2}\right)\right. \\
& -\frac{176}{105} v_{1}+1 \leftrightarrow 2 .
\end{aligned}
$$

and

$$
\begin{aligned}
\mathbf{G}^{2.5 \mathrm{PN}}= & \frac{4 G m_{1} m_{2}}{5 c^{5}}\left(v_{12}^{2}-\frac{2 G\left(m_{1}+m_{2}\right)}{r_{12}}\right) \boldsymbol{v}_{1}+1 \leftrightarrow 2 \\
\mathbf{G}^{3.5 \mathrm{PN}}= & \left\{-\frac{8}{3} \frac{G^{3} m_{1}^{3} m_{2}}{r_{12}^{2}}\left(n_{12} v_{1}\right)+\frac{24}{35} \frac{G^{3} m_{1}^{2} m_{2}^{2}}{r_{12}^{2}}\left(n_{12} v_{1}\right)+\frac{52}{15} \frac{G^{3} m_{1} m_{2}^{3}}{r_{12}^{2}}\left(n_{12} v_{1}\right)\right. \\
& +\frac{G^{2} m_{1} m_{2}^{2}}{r_{12}}\left(-\frac{8}{5}\left(n_{12} v_{1}\right)^{3}+\frac{24}{5}\left(n_{12} v_{1}\right)^{2}\left(n_{12} v_{2}\right)+\frac{4}{15}\left(n_{12} v_{1}\right)\left(v_{1} v_{2}\right)\right. \\
& \left.-\frac{2}{15}\left(n_{12} v_{1}\right) v_{1}^{2}-\frac{4}{15}\left(n_{12} v_{2}\right) v_{1}^{2}\right) \\
& +\frac{G^{2} m_{1}^{2} m_{2}}{r_{12}}\left(\frac{8}{5}\left(n_{12} v_{1}\right)^{3}-\frac{24}{5}\left(n_{12} v_{1}\right)^{2}\left(n_{12} v_{2}\right)+\frac{8}{15}\left(n_{12} v_{1}\right)\left(v_{1} v_{2}\right)\right. \\
& \left.\left.-\frac{76}{105}\left(n_{12} v_{1}\right) v_{1}^{2}-\frac{2}{15}\left(n_{12} v_{2}\right) v_{1}^{2}\right)\right\} \boldsymbol{n}_{12} \\
& +\left\{-\frac{4}{15} \frac{G^{3} m_{1}^{2} m_{2}^{2}}{r_{12}^{2}}-\frac{172}{105} \frac{G^{3} m_{1} m_{2}^{3}}{r_{12}^{2}}+\frac{34}{21} \frac{G^{2} m_{1}^{3}}{r_{12}} v_{1}^{2}\right.
\end{aligned}
$$




$$
\begin{aligned}
& +\frac{G^{2} m_{1} m_{2}^{2}}{r_{12}}\left(\frac{44}{15}\left(n_{12} v_{1}\right)^{2}-\frac{88}{15}\left(n_{12} v_{1}\right)\left(n_{12} v_{2}\right)+\frac{56}{15}\left(n_{12} v_{2}\right)^{2}\right. \\
& \left.\quad-\frac{68}{35}\left(v_{1} v_{2}\right)+\frac{6}{35} v_{1}^{2}+\frac{188}{105} v_{2}^{2}\right) \\
& +\frac{G^{2} m_{1}^{2} m_{2}}{r_{12}}\left(-\frac{104}{105}\left(n_{12} v_{1}\right)^{2}+\frac{88}{15}\left(n_{12} v_{1}\right)\left(n_{12} v_{2}\right)-\frac{44}{15}\left(n_{12} v_{2}\right)^{2}\right. \\
& \left.\quad-\frac{1144}{105}\left(v_{1} v_{2}\right)+\frac{634}{105} v_{1}^{2}+\frac{118}{15} v_{2}^{2}\right) \\
& +G m_{1}^{2}\left(\frac{34}{21}\left(v_{1} v_{2}\right) v_{1}^{2}-\frac{13}{15} v_{1}^{4}-\frac{17}{21} v_{1}^{2} v_{2}^{2}\right) \\
& +G m_{1} m_{2}\left(-\frac{4}{7}\left(v_{1} v_{2}\right)^{2}-\frac{36}{35}\left(v_{1} v_{2}\right) v_{1}^{2}+\frac{6}{7} v_{1}^{4}+\frac{22}{21}\left(v_{1} v_{2}\right) v_{2}^{2}\right. \\
& \left.\left.+\frac{11}{105} v_{1}^{2} v_{2}^{2}-\frac{7}{15} v_{2}^{4}\right)\right\} \boldsymbol{v}_{1} \\
& +\left\{-\frac{4}{5} \frac{G^{3} m_{1}^{3} m_{2}}{r_{12}^{3}}\left(n_{12} v_{1}\right)+\frac{G^{3} m_{1}^{2} m_{2}^{2}}{r_{12}^{3}}\left(\frac{4}{5}\left(n_{12} v_{1}\right)-\frac{4}{5}\left(n_{12} v_{2}\right)\right)+\frac{4}{5} \frac{G^{3} m_{1} m_{2}^{3}}{r_{12}^{3}}\left(n_{12} v_{2}\right)\right. \\
& +\frac{G^{2} m_{1}^{2} m_{2}}{r_{12}^{2}}\left(-\frac{4}{5}\left(n_{12} v_{1}\right)\left(v_{1} v_{2}\right)+\frac{2}{5}\left(n_{12} v_{1}\right) v_{1}^{2}+\frac{2}{5}\left(n_{12} v_{1}\right) v_{2}^{2}\right) \\
& \left.+\frac{G^{2} m_{1} m_{2}^{2}}{r_{12}^{2}}\left(\frac{4}{5}\left(n_{12} v_{2}\right)\left(v_{1} v_{2}\right)-\frac{2}{5}\left(n_{12} v_{2}\right) v_{1}^{2}-\frac{2}{5}\left(n_{12} v_{2}\right) v_{2}^{2}\right)\right\} \boldsymbol{y}_{1}+1 \leftrightarrow 2 .
\end{aligned}
$$

As we said, showing the existence of these 2.5PN and 3.5PN contributions to the linear momentum and center of mass constitutes a full proof of the balance equations (3.9) and (3.17). Note that there is no point about trying to relate theses terms to those, given by Eqs. (3.11) and (3.19), obtained in the more general investigation of Sec. III. Indeed, the calculation done in Sec. III used the extended Burke-Thorne coordinate system [22] while the present calculation employs harmonic coordinates.

We pospone to further work the discussion of the radiation-reaction terms in the energy $E$ and angular momentum $J_{i}$ in a general frame. Note that these have already been given in the frame of the center of mass by Iyer \& Will [26, 27] and, in the harmonic gauge, by Ref. [62]; see Eqs. (6.7) and (6.9) there.

\section{DISCUSSION AND CONCLUSION}

We have obtained the equations for the secular evolution by GW emission of the linear momentum $\boldsymbol{P}$ and center-of-mass position $\boldsymbol{G}$ of an isolated post-Newtonian source,

$$
\begin{aligned}
& \frac{\mathrm{d} \boldsymbol{P}}{\mathrm{d} t}=-\boldsymbol{F}_{P}, \\
& \frac{\mathrm{d} \boldsymbol{G}}{\mathrm{d} t}=\boldsymbol{P}-\boldsymbol{F}_{G},
\end{aligned}
$$

where $\boldsymbol{F}_{P}$ and $\boldsymbol{F}_{G}$ represent the fluxes given by (1.3) and (1.4), or more generally by (4.17b) and $(4.18 \mathrm{~b})$. Let us now consider the case, to start with, where the source is stationary before some instant $t_{0}$, then emits a pulse of gravitational waves with finite duration between times $t_{0}$ and $t_{1}$, and finally comes back to a stationary state at later times $t>t_{1}$. This means that the fluxes $\boldsymbol{F}_{P}$ and $\boldsymbol{F}_{G}$ are zero outside the time of emission, when $t<t_{0}$ and $t>t_{1}$. 
In this situation, it is straightforward to find the form of the solution to Eqs. (6.1). Initially, the linear momentum is constant, so that, by applying a Lorentz boost, we can put ourselves in the rest frame of the source, thus achieving $\boldsymbol{P}_{0}=\mathbf{0}$ (for $t<t_{0}$ ). Furthermore, we can translate the origin of our coordinate system in such a way that it coincides with the center of mass of the source, hence $\boldsymbol{G}_{0}=\mathbf{0}$ initially. Then, by integrating (6.1), we get (for $\left.t_{0}<t<t_{1}\right)$

$$
\begin{aligned}
& \boldsymbol{P}(t)=-\int_{t_{0}}^{t} \mathrm{~d} t^{\prime} \boldsymbol{F}_{P}\left(t^{\prime}\right), \\
& \boldsymbol{G}(t)=-\int_{t_{0}}^{t} \mathrm{~d} t^{\prime}\left(t-t^{\prime}\right) \boldsymbol{F}_{P}\left(t^{\prime}\right)-\int_{t_{0}}^{t} \mathrm{~d} t^{\prime} \boldsymbol{F}_{G}\left(t^{\prime}\right) .
\end{aligned}
$$

After the period of emission (for $t>t_{1}$ ), the source is again stationary but has acquired a net constant linear momentum $\boldsymbol{P}_{1}$ with respect to its initial value $\boldsymbol{P}_{0}=\mathbf{0}$ and the motion of its center of mass has become uniform, i.e. $\boldsymbol{G}_{1}=\boldsymbol{P}_{1} t+\boldsymbol{Z}_{1}$. We find

$$
\begin{aligned}
& \boldsymbol{P}_{1}=-\int_{t_{0}}^{t_{1}} \mathrm{~d} t^{\prime} \boldsymbol{F}_{P}\left(t^{\prime}\right), \\
& \boldsymbol{Z}_{1}=\int_{t_{0}}^{t_{1}} \mathrm{~d} t^{\prime}\left[t^{\prime} \boldsymbol{F}_{P}\left(t^{\prime}\right)-\boldsymbol{F}_{G}\left(t^{\prime}\right)\right] .
\end{aligned}
$$

As we see, the cumulative effect of the flux $\boldsymbol{F}_{G}$ results in adding an extra contribution to the position of the center of mass after the GW emission. On the other hand, the final value of the linear momentum $\boldsymbol{P}_{1}$ provides the total gravitational recoil velocity of the source (or kick), as measured in the asymptotic Minkowskian frame:

$$
\boldsymbol{V}_{1}=\frac{\boldsymbol{P}_{1}}{\sqrt{M_{1}^{2}+\frac{\boldsymbol{P}_{1}^{2}}{c^{2}}}},
$$

where $M_{1}$ is the final mass of the system, after the GW emission has stopped. The variation of the mass is obtained by integrating the energy balance equation,

$$
M_{1}=M_{0}-\frac{1}{c^{2}} \int_{t_{0}}^{t_{1}} \mathrm{~d} t^{\prime} F_{E}\left(t^{\prime}\right),
$$

where $F_{E}$ is the energy flux, for instance given by (1.1). However, since the fluxes represent small quantities in the adiabatic approximation, the mass $M_{1}$ under the square root of Eq. (6.4) might be approximated by the initial value $M_{0}{ }^{11}$ whereas the relativistic correction $\boldsymbol{P}_{1}^{2} / c^{2}$ can be neglected. The gravitational recoil (6.4) has been investigated for general systems in [28-32] and some estimations have been proposed for binary systems in [33-38].

We consider next the "instantaneous" motion of the center of mass during the GW emission, as given by Eqs. (6.2). To be specific, we focus on the case of a Newtonian binary system with no spins and moving on an exactly circular orbit (we neglect the radiation reaction on the orbit). We introduce the symmetric mass ratio $\nu=m_{1} m_{2} / m^{2}$ with $m=$

${ }^{11}$ Numerical relativity (NR) calculations show that the energy radiated away by black-hole binary systems represents a few percents of their total mass energy; see, e.g., Fig. 9 of Ref. [46] (top right panel). 
$m_{1}+m_{2}$, assuming $m_{1} \geqslant m_{2}$ so that $m_{1}-m_{2}=m \sqrt{1-4 \nu}$. The orbital separation is denoted by $r$ and the unit vector along the binary's separation (pointing towards the larger mass $m_{1}$ ) by $\boldsymbol{n}$. We also define the unit vector $\boldsymbol{\lambda}$ orthogonal to $\boldsymbol{n}$ in the orbital plane and oriented in the sense of the relative motion. For circular orbits, the relative velocity of the particles reduces to $\boldsymbol{v}=r \boldsymbol{\omega} \boldsymbol{\lambda}$, where $\omega=\sqrt{G m / r^{3}}$ is the (Newtonian) orbital frequency.

The RHS of the linear momentum flux equation (3.9) is straightforward to evaluate for the Newtonian circular binary system with result [33]

$$
\frac{\mathrm{d} \boldsymbol{P}}{\mathrm{d} t}=\frac{464}{105} \frac{G^{4} m^{5} \omega}{c^{7} r^{4}} \sqrt{1-4 \nu} \nu^{2} \boldsymbol{\lambda}
$$

This relation holds at any time along the orbit and can be integrated, yielding

$$
\boldsymbol{P}=\frac{464}{105} \frac{G^{4} m^{5}}{c^{7} r^{4}} \sqrt{1-4 \nu} \nu^{2} \boldsymbol{n}
$$

where we assume from now on that a Lorentz boost and a shift of the origin of the coordinate system have been applied to set $\boldsymbol{P}$ and $\boldsymbol{G}$ to zero when averaged over an orbit (neglecting the radiation-reaction decay). Then, we use the center-of-mass balance equation (3.17), which leads to

$$
\frac{\mathrm{d} \boldsymbol{G}}{\mathrm{d} t}=\boldsymbol{P}+\frac{544}{105} \frac{G^{4} m^{5}}{c^{7} r^{4}} \sqrt{1-4 \nu} \nu^{2} \boldsymbol{n},
$$

so that, combining the two previous results and integrating,

$$
\boldsymbol{G}=-\frac{48}{5} \frac{G^{4} m^{5}}{c^{7} r^{4} \omega} \sqrt{1-4 \nu} \nu^{2} \boldsymbol{\lambda} .
$$

The equations (6.7) and (6.9) give the instantaneous values of the momentum and centerof-mass position of a circular binary system (neglecting the orbital decay). Their RHS are equal to minus the values that can be attributed to the gravitational radiation field. It would be interesting to compare the prediction (6.9) for the oscillations of the center of mass with very accurate numerical computations of the recoil and center-of-mass position, such as those of Ref. [46]. Perhaps the problem, in performing such comparison, would be the control of the different gauges used by PN and NR calculations.

To conclude, we have argued that the usual "quadrupole-type" formulas for the energy, angular momentum and linear momentum, are missing an analogous formula for the position of the center of mass. Indeed, the complete set of invariants of a relativistic system does include the (initial position of the) center of mass, which is the Noetherian quantity associated with the invariance of the dynamics under Lorentz boosts. We have found three derivations of the balance equation describing the effect of GW emission on the position of the center of mass. The first one is based on the local equations of motion of a general isolated post-Newtonian source, including the gravitational radiation-reaction force at the 3.5PN order in a specific gauge, which have been integrated over the volume of the source, leading to the requested flux equation [see Secs. II and III]. The second derivation, also valid for a general isolated source, is a direct flux calculation performed at future null infinity, yielding the full multipole moment expansion for the flux, though restricted to the dominant post-Minkowskian order [see Sec. IV]. Finally, we have verified that, for the known radiation-reaction force at the $3.5 \mathrm{PN}$ order in the particular case of compact binary systems, the balance equation is indeed satisfied [see Sec. V]. Independent and complementary derivations of the balance equation for the center-of-mass position have been achieved recently [59, 61]. Further work is needed to know whether there could be any possible interest and/or application for this effect in Astrophysics. 


\section{Acknowledgments}

We are grateful to Clifford Will and Alexandre Le Tiec for interesting discussions. More-

over, we appreciated the comments made on a first version of this paper by Bala Iyer, Carlos Kozameh, David Nichols and Leo Stein.

[1] R. Hulse and J. Taylor, Astrophys. J. 195, L51 (1975).

[2] J. Ehlers, A. Rosenblum, J. Goldberg, and P. Havas, Astrophys. J. 208, L77 (1976).

[3] M. Walker and C. Will, Astrophys. J. 242, L129 (1980).

[4] S. Chandrasekhar and F. Esposito, Astrophys. J. 160, 153 (1970).

[5] W. Burke, J. Math. Phys. 12, 401 (1971).

[6] W. Burke and K. Thorne, in Relativity, edited by M. Carmeli, S. Fickler, and L. Witten (Plenum Press, New York and London, 1970), pp. 209-228.

[7] G. Kerlick, Gen. Relativ. Gravit. 12, 467 (1980).

[8] G. Kerlick, Gen. Relativ. Gravit. 12, 521 (1980).

[9] A. Papapetrou and B. Linet, Gen. Relativ. Gravit. 13, 335 (1981).

[10] A. Einstein, Sitzber. Preuss. Akad. Wiss. Berlin 1, 154 (1918).

[11] L. Landau and E. Lifshitz, The classical theory of fields (Pergamon, Oxford, 1971).

[12] P. Peters and J. Mathews, Phys. Rev. 131, 435 (1963).

[13] P. Peters, Phys. Rev. 136, B1224 (1964).

[14] L. Esposito and E. Harrison, Astrophys. J. 196, L1 (1975).

[15] R. Wagoner, Astrophys. J. 196, L63 (1975).

[16] T. Damour, in Gravitational Radiation, edited by N. Deruelle and T. Piran (North-Holland Company, Amsterdam, 1983), pp. 59-144.

[17] T. Damour, Phys. Rev. Lett. 51, 1019 (1983).

[18] L. Blanchet and T. Damour, Phys. Rev. D 37, 1410 (1988).

[19] L. Blanchet and T. Damour, Annales Inst. H. Poincaré Phys. Théor. 50, 377 (1989).

[20] L. Blanchet and T. Damour, Phys. Rev. D 46, 4304 (1992).

[21] L. Blanchet, Phys. Rev. D 47, 4392 (1993).

[22] L. Blanchet, Phys. Rev. D 55, 714 (1997), gr-qc/9609049.

[23] L. Blanchet, Living Rev. Rel. 17, 2 (2014), arXiv:1310.1528 [gr-qc].

[24] A. Buonanno and B. Sathyaprakash, in General Relativity and Gravitation: A Centennial Perspective, edited by A. Ashtekar, B. Berger, J. Isenberg, and M. MacCallum (2015), p. 513, arXiv:1410.7832 [gr-qc].

[25] G. Schott, Phil. Mag. 29, 49 (1915).

[26] B. Iyer and C. Will, Phys. Rev. Lett. 70, 113 (1993).

[27] B. Iyer and C. Will, Phys. Rev. D 52, 6882 (1995).

[28] A. Papapetrou, Ann. Inst. Henri Poincaré XIV, 79 (1971).

[29] K. Thorne, Rev. Mod. Phys. 52, 299 (1980).

[30] W. Bonnor and M. Rotenberg, Proc. R. Soc. London, Ser. A 265, 109 (1961).

[31] A. Peres, Phys. Rev. 128, 2471 (1962).

[32] J. Bekenstein, Astrophys. J. 183, 657 (1973).

[33] M. J. Fitchett, Mon. Not. Roy. Astron. Soc. 203, 1049 (1983).

[34] A. G. Wiseman, Phys. Rev. D 46, 1517 (1992). 
[35] L. Kidder, Phys. Rev. D 52, 821 (1995), gr-qc/9506022.

[36] L. Blanchet, M. S. Qusailah, and C. M. Will, Astrophys. J. 635, 508 (2005), astro-ph/0507692.

[37] E. Racine, A. Buonanno, and L. Kidder, Phys. Rev. D 80, 044010 (2009), arXiv:0812.4413 [gr-qc].

[38] A. Le Tiec, L. Blanchet, and C. Will, Class. Quant. Grav. 27, 012001 (2010), arXiv:0901.4594 [gr-qc].

[39] K. Oohara and T. Nakamura, Prog. Theor. Phys. 70, 757 (1983).

[40] M. J. Fitchett and S. Detweiler, Mon. Not. Roy. Astron. Soc. 211, 933 (1984).

[41] T. Nakamura, K. Oohara, and Y. Kojima, Prog. Theor. Phys. Suppl. 90, 1 (1987).

[42] M. Favata, S. A. Hughes, and D. E. Holz, Astrophys. J. Lett. 607, L5 (2004), astro$\mathrm{ph} / 0402056$.

[43] M. Campanelli, Class. Quant. Grav. 22, S387 (2005), astro-ph/0411744.

[44] J. G. Baker, J. Centrella, D.-I. Choi, M. Koppitz, J. van Meter, and M. Miller, Astrophys. J. 653, L93 (2006), astro-ph/0603204.

[45] M. Campanelli, C. O. Lousto, Y. Zwlochower, and D. Merritt, Astrophys. J. 659, L5 (2007), gr-qc/0701164.

[46] D. Gerosa, F. Hébert, and L. Stein, Phys. Rev. D 97, 104049 (2018), arXiv:1802.04276 [gr-qc].

[47] H. Bondi, M. van der Burg, and A. Metzner, Proc. R. Soc. London, Ser. A 269, 21 (1962).

[48] R. Sachs, Proc. R. Soc. London, Ser. A 270, 103 (1962).

[49] R. Arnowitt, S. Deser, and C. Misner, in An introduction to current research, edited by L. Witten (New York: Wiley, 1962).

[50] C. Misner, K. Thorne, and J. Wheeler, Gravitation (Freeman, San Francisco, 1973).

[51] S. Weinberg, Gravitation and Cosmology (John Wiley, New York, 1972).

[52] R. Wald, General Relativity (University of Chicago Press, 1984).

[53] M. Maggiore, Gravitational waves (Oxford University Press, 2008).

[54] E. Poisson and C. Will, Gravity: Newtonian, post-Newtonian, relativistic (Cambridge University Press, 2014).

[55] K. Thorne, Gravitational Radiation (Cambridge University Press, 1987), pp. 330-458.

[56] T. Damour, The problem of motion in Newtonian and Einsteinian gravity (Cambridge University Press, 1987), pp. 128-198.

[57] T. Damour, in Gravitation in Astrophysics. Cargèse 1986, edited by B. Carter and J. Hartle (Plenum Press, New York and London, 1986), pp. 3-62.

[58] C. Handmer, B. Szilágyi, and J. Winicour, Class. Quant. Gravity 33, 225007 (2016), arXiv:1605.04332 [gr-qc].

[59] C. Kozameh, J. Nieva, and G. Quirega, Phys. Rev. D 98, 064032 (2018), arXiv:1711.11375 [gr-qc].

[60] C. Kozameh and G. Quirega, Phys. Rev. D 93, 064050 (2016), arXiv:1311.5854 [gr-qc].

[61] D. Nichols, Phys. Rev. D 98, 064032 (2018), arXiv:1807.08767 [gr-qc].

[62] L. Bernard, L. Blanchet, G. Faye, and T. Marchand, Phys. Rev. D 97, 044037 (2018), arXiv:1711.00283 [gr-qc].

[63] B. Bonga and E. Poisson (2018), arXiv:1808.01288 [gr-qc].

[64] P. Jaranowski and G. Schäfer, Phys. Rev. D 55, 4712 (1997).

[65] M. Pati and C. Will, Phys. Rev. D 65, 104008 (2002), gr-qc/0201001.

[66] C. Königsdörffer, G. Faye, and G. Schäfer, Phys. Rev. D 68, 044004 (2003), astro-ph/0305048.

[67] S. Nissanke and L. Blanchet, Class. Quant. Grav. 22, 1007 (2005), gr-qc/0412018.

[68] Y. Itoh, Phys. Rev. D 80, 024003 (2009), arXiv:0911.4232 [gr-qc]. 